\title{
Numerical simulation of an oscillating cylinder in a cross-flow at low Reynolds number: Forced and free oscillations
}

\author{
Antoine Placzek $^{a}$, Jean-François Sigrist ${ }^{\mathrm{b}, *}$, Aziz Hamdouni ${ }^{\mathrm{c}}$ \\ ${ }^{a}$ ONERA, The French Aerospace Lab, Aeroelasticity and Structural Dynamics Department, 29 Avenue de la Division Leclerc, \\ BP72, 92322 Châtillon Cedex, France \\ ${ }^{\mathrm{b}}$ Service Scientifique et Technique, DCNS Propulsion, 44620 La Montagne, France \\ ${ }^{\mathrm{c}}$ Laboratoire d'Étude des Phénomènes de Transferts Appliqueś au Bâtiment, Université de La Rochelle, Avenue Michel Crépeau, \\ 17042 La Rochelle Cedex 1, France
}

\begin{abstract}
A numerical simulation of the flow past a circular cylinder which is able to oscillate transversely to the incident stream is presented in this paper for a fixed Reynolds number equal to 100. The 2D Navier-Stokes equations are solved by a finite volume method with an industrial CFD code in which a coupling procedure has been implemented in order to obtain the cylinder displacement. A preliminary work is first conducted for a fixed cylinder to check the wake characteristics for Reynolds numbers smaller than 150 in the laminar regime. The Strouhal frequency $f_{\mathrm{S}}$ and the aerodynamic coefficients are thus controlled among other parameters. Simulations are then performed with forced oscillations characterized by the frequency ratio $F=f_{0} / f_{\mathrm{S}}$, where $f_{0}$ is the forced oscillation frequency, and by the adimensional amplitude $A$. The wake characteristics are analyzed using the ti me series of the fluctuating aerodynamic coefficients and their power spectral densities (PSD). The frequency content is then linked to the shape of the phase portraits and to the vortex shedding mode. By choosing interesting couples $(A, F)$, different vortex shedding modes have been observed, which are similar to those of the Wil-liamsonRoshko map. A second batch of simulations involving free vibrations (so-called vortex-induced vibrations or VIV) is finally carried out. Oscillations of the cylinder are now directly induced by the vortex shedding process in the wake and therefore, the time inte-gration of the motion is realized by an explicit staggered algorithm which provides the cylinder displacement according to the aerody-namic charges exerted on the cylinder wall. Amplitude and frequency response of the cylinder are thus investigated over a wide range of reduced velocities to observe the different phenomena at stake. In particular, the vortex shedding modes have also been related to the frequency response observed and our results at $R e=100$ show a very good agreement with other studies using different numerical approaches.
\end{abstract}

\section{Introduction}

Flow around a fixed or oscillating cylinder has received continued attention in the past few decades. In addition to being a building block in the understanding of bluff body dynamics, it has a large number of applications in many engineering situations. This study is the first step to investigate the feasibility of coupled fluid-structure computa-

\footnotetext{
* Corresponding author. Tel.: +332408487 84; fax: +33240848718. E-mail address: jean-francois.sigrist@densgroup.com (J.-F. Sigrist).
}

tions with an industrial CFD code which could be used later on a tube bundle configuration, like those existing in nuclear steam generators.

The wake of a fixed circular cylinder exhibits a large variety of complex phenomena stemming from the diverse instabilities growing in the near wake. The classification of these phenomena was primarily based on experimental measurements and therefore the limits describing the transition between the different regimes were sometimes not exactly established. However a rather clear classification relying either on the evolution of the Strouhal number [8] or on 
the base pressure coefficient curve [48] is nowadays available. According to these classifications, the following regimes can be highlighted: for $R e \lesssim 49$, two stationary recirculation zones attached to the cylinder wall can be observed; then for $49 \lesssim R e \lesssim 190$, the wake is still laminar and consists of two periodic staggered rows of vortices forming the well-known Von Kármán streets, the vortices of each row being shed alternately from either side of the cylinder. For greater Reynolds numbers, the wake becomes three-dimensional (for $190 \lesssim R e \lesssim 260$ ) and progressively turbulent. This regime is followed by the shear-layer transition $(R e \gtrsim 1200)$ where the separating shear layers become unstable and finally by the boundary-layer transition $(R e$ of order $10^{5}$ ) which is associated to the drag crisis, i.e. a dramatic decrease of the drag coefficient. Over all these regimes, the flow exhibits a certain periodicity which is known as the Strouhal frequency, denoted here by $f_{\mathrm{S}}$. When a periodic vortex street is well established, this frequency corresponds to that of the vortex shedding frequency; in other cases where the Von Kármán streets are not clearly visible, the frequency can be defined as the one of the fluctuations of the streamwise velocity component for example.

In many applications, the cylinder is not fixed but oscillates at a given frequency that could interact with the vortex shedding process. For forced oscillations in a certain range of amplitude and frequency, the cylinder motion is able to control the instability mechanism which leads to vortex shedding. One of the most interesting characteristics of this fluid-structure interaction is the synchronization, or "lock-in", between the vortex shedding and vibration frequencies. The vortex shedding frequency diverges from that corresponding to a fixed cylinder $f_{\mathrm{S}}$ and becomes equal to the forced oscillation frequency $f_{0}$. Similar phenomena are observed for vortex-induced vibrations (VIV): in this case, the flow causes the cylinder to oscillate at its natural frequency $f_{\mathrm{N}}$ which depends on the mass, the rigidity and possibly the damping of the cylinder. The cylinder oscillation frequency is thus different from the Strouhal frequency $f_{\mathrm{S}}$ that would be obtained if the cylinder was supposed to be fixed. This phenomenon occurs over a certain range of reduced velocities, where the vortex shedding frequency becomes equal to the natural frequency and a peak of amplitude is reached.

This complicated fluid-structure interaction phenomenon still draws the attention of researchers and has become the typical test case for new numerical techniques. A lot of studies, involving Reynolds Averaged Navier-Stokes (RANS) methods [39,18], Large Eddy Simulations (LES) [6,32,2,15], Direct Numerical Simulations (DNS) [11], using finite volume or finite element $[3,29,27]$ approximations to solve the Navier-Stokes equations, can be found in the literature for a large range of Reynolds numbers. It is also crucial to check that the numerical computation leads to the same phenomena than those observed in experimental works $[7,21]$.

The present study aims at performing numerical simulations of VIV using a general numerical tool that can be fur- ther used to study industrial problems using a general approach based on computational fluid dynamics and computational solid dynamics code coupling. It is therefore of paramount importance to validate the fluid code and the coupling procedure for such applications prior to perform numerical studies on real configurations. Validation is achieved by comparing the numerical results of our VIV simulations with other numerical studies and discussing the observed simulated phenomena. The paper is organized as follows: the numerical model used for the computations is first presented; numerical results obtained for fluid flow around a fixed cylinder are then briefly exposed before presenting the structure response and fluid flow pattern for forced and free oscillations of the cylinder.

\section{Numerical model}

\subsection{Resolution of the Navier-Stokes equations}

The flow field is governed by the Navier-Stokes equations, which read for a Newtonian incompressible fluid:

$$
\left\{\begin{array}{l}
\operatorname{div} \mathbf{u}=\mathbf{0} \\
\frac{\partial \mathbf{u}}{\partial t}+\operatorname{div}(\mathbf{u} \otimes \mathbf{u})=-\frac{1}{\rho} \nabla p+v \Delta \mathbf{u}
\end{array}\right.
$$

where $\mathbf{u}=(u v)^{\mathrm{T}}$ is the velocity vector with $u$ and $v$ being respectively the streamwise and transverse velocity components, $p$ is the pressure, $\rho$ and $v$ are the fluid density and kinematic viscosity. As the Reynolds number does not rise above 190 , the flow is assumed to be laminar and two-dimensional according to the description of the flow regimes given by Williamson [48] for this range of Reynolds numbers.

The Navier-Stokes equations are discretized using the finite volume technique, i.e. integral form of the conservation equations are solved on control volumes which form a partition of the computational domain (see [13] for more details). Surface and volume integral approximations require the values of variables at locations other than the computational nodes. Indeed, the integrand involves the product of several variables (convective fluxes) or variable gradients at those locations (diffusive fluxes). Interpolations are therefore performed to evaluate these fluxes with the high-order MARS (Monotone Advection and Reconstruction Scheme) algorithm which has been developed especially for the CFD code used here [46]. The MARS algorithm is a second order conditional upwinding approximation developed in order to decrease the numerical dispersion. For unsteady computations, the time dependent term is approximated by an Euler scheme (implicit scheme).

The pressure-velocity decoupling is achieved by the SIMPLE (Semi-Implicit Method for Pressure-Linked Equations) algorithm for steady computations or the PISO (pressure implicit with splitting of operators) procedure for unsteady cases. The pressure field is first predicted, then corrected by several iterations so that the Poisson equation 
for pressure and the momentum conservation equations for velocity are satisfied.

Once Eqs. (1) have been discretized and the pressurevelocity decoupling has been realized, the problem is represented by a matrix system composed of the cell-centered unknowns which has to be inverted. The resolution is performed with the pre-conditioned conjugate gradient method and provides the velocity components $u$ and $v$, and the pressure $p$. The same CFD code and numerical techniques have already been used by Sigrist and Abouri [42]. A complementary description of the whole resolution method can also be found in this reference.

\subsection{Geometry and boundary conditions}

The computational domain is represented in Fig. 1a with the cylinder of diameter $d$. The size of the domain and the position of the cylinder have been chosen according to simulations performed by Gerouache [16] with the same code for different lengths and heights. The results are very sensitive to the size of the computational domain, particularly when the Reynolds number is small. Indeed, as the Reynolds number decreases, the flow is mainly governed by the viscous effects whose region of influence varies with $R e^{-1}$. If the computational domain is not chosen wide enough to contain this influence region, the error caused by the artificial boundary conditions disturbs the solution, even near the cylinder. For $R e<1$, Lange et al. [25] advise choosing $H / D>320 R e^{-0.8}$ to maintain the error smaller than $1 \%$. For greater Reynolds numbers [33] showed that the aspect ratio should not be smaller than $H / d=22$ for $R e=100$ and that the influence of the outlet boundary condition becomes negligible for $L_{2} / d \geqslant 34$. The size of the computational domain has been chosen here to keep the number of control cells to a reasonable amount, although the length and height are not conform to the values advocated by [33]. However, the configuration retained $\left(H / d=20, L_{2} / d=20\right)$ provides acceptable results and the authors are aware of the blockage effects, particularly as

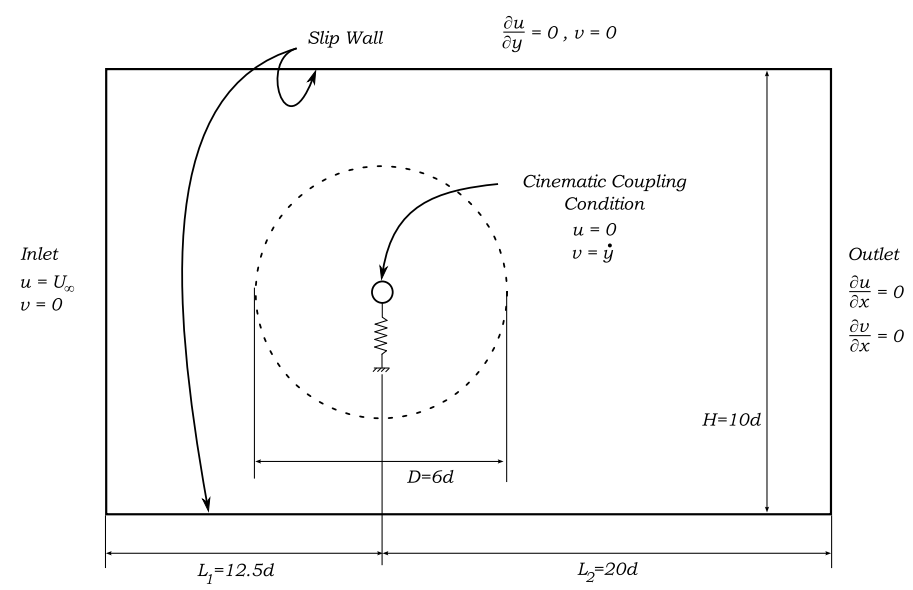

(a) Size and geometric disposition of the computational domain regards the surestimation of the drag coefficient and Strouhal number when the Reynolds number is small.

The mesh presented in Fig. 1b is block-structured and a ring of diameter $D=12 d$ (represented with a dashed line in Fig. 1a) has been introduced around the cylinder to facilitate the use of the moving mesh procedure when the cylinder oscillates. Grid independence tests have already been performed by Gerouache [16] for the same configuration and CFD code. The configuration of the blocks and the number of control cells have therefore been built according to the observations made in this study. Thus the total number of cells in the whole model used here stands at 28,800. It should also be mentioned that a ratio has been introduced along the radial direction in the ring surrounding the cylinder, so that the mesh is finer near the cylinder wall and becomes coarser away from it (see Fig. 1b).

The boundary conditions adopted are specified in Fig. 1a. The inlet velocity $U_{\infty}$ is chosen according to the cylinder diameter $d$ and the fluid characteristics $(\rho, v)$ to obtain the desired Reynolds number: $R e=U_{\infty} d / v$. When the cylinder is moving, particular attention should be paid to respect the cinematic coupling condition at the cylinder wall. Continuity of the velocities imposes the following equality for an unidirectional vertical motion:

$v=\dot{y} \quad$ on $\Gamma$

where $v$ is the vertical component of the fluid velocity, $\dot{y}$ is the cylinder velocity and $\Gamma$ denotes the cylinder wall. In order to reduce the computational time, the moving mesh procedure has been developed as a user subroutine so that it operates only in the ring surrounding the cylinder and leads to a deformed mesh whose structure is preserved during the oscillations [41].

\subsection{Cylinder motion}

Two different techniques will be used in this study to obtain the cylinder motion. On the one hand, when forced

Fig. 1. Configuration of the computational domain and overview of the mesh used for the simulations. $d$ is the cylinder diameter, $x$ is the horizontal direction, $y$ the vertical one. 
oscillations are imposed, the motion is known and can therefore be directly imposed. On the other hand, when the motion results from the vortex shedding process, the displacement has to be computed from the fluid forces before it could be applied to the cylinder.

The forced oscillations of the cylinder are characterized by the frequency $f_{0}$ and the maximal adimensional amplitude $A=y_{\max } / d$, where $y_{\max }$ is the maximal vertical imposed displacement. A sinusoidal motion governed by the following equation is explicitly imposed to the cylinder in a dedicated subroutine implemented within the CFD code at each time step before solving the flow field:

$y(t)=y_{\max } \sin \left(2 \pi f_{0} t\right)$

The cylinder oscillates independently from the flow but the wake can be strongly affected by the cylinder motion. The different wake regimes are classified thanks to the adimensional amplitude $A$ defined above and the frequency ratio $F=f_{0} / f_{\mathrm{S}}$, where $f_{\mathrm{S}}$ refers to the Strouhal frequency for the fixed cylinder.

When vortex-induced vibrations are studied, the motion corresponds generally to the cylinder bending mode. In two-dimensions, the cylinder flexibility can be easily modeled by a mass-spring system excited by the fluid forces. The vertical cylinder motion $y(t)$ is consequently governed by the equation of the following undamped oscillator:

$m \ddot{y}+k y=F_{y}$

where $m$ denotes the cylinder mass, $k$ is the rigidity of the fictitious spring and $F_{\mathrm{y}}$ is the resultant of the lift force (vertical component of the aerodynamic force), this latter being a priori unknown. In absence of external forces, Eq. (4) provides the natural cylinder frequency $f_{\mathrm{N}}$ which will be used later to drive the oscillations:

$f_{\mathrm{N}}=\frac{1}{2 \pi} \sqrt{\frac{k}{m}}$

The couple $(m, k)$ could thus be chosen to represent the frequency of the bending mode, but when fluid forces are not null, the actual oscillation frequency $f$ depends on them and is consequently different from $f_{\mathrm{N}}$ in general. The resolution of Eq. (4) requires the knowledge of the lift force $F_{\mathrm{y}}(t)$, meaning that the flow field has to be computed before. In fact, this is a typical fluid-structure interaction problem because the lift force influences the cylinder displacement $y(t)$ which in turn modifies the flow field and therefore $F_{\mathrm{y}}(t)$, and so on.

The resolution of such problems can be conducted relatively easily by using staggered procedures, i.e. the fluid and the structure are solved successively for a given time step. A detailed description of such algorithms has been proposed for example by Piperno [34] or Farhat et al. [12]. Unfortunately, these methods lead inevitably to a time shift between the fluid and the structure, which are not computed exactly at the same time step. The main drawback is that the coupling rely on a prediction of the displacement which has to be as accurate as possible, otherwise the energy transfer at the fluid-structure interface is not well evaluated. As a consequence, the coupling procedure is likely to cause artificial instabilities (due for example to a negative numerical damping) leading to the divergence of the system. This numerical problem can be by-passed with the use of implicit procedures introducing subiterations for the same time step and leading to the convergence of the displacement and fluid force at a given time step (see examples in $[1,17,23,44])$. Implicit coupling has been used for instance by Sigrist and Abouri [42] who have noticed its superiority for non-linear coupled problems (shock responses). Comparisons between different coupling procedures (implicit and explicit) have also been performed by Placzek et al. [36] on a confined cylinder configuration to chose the best compromise between CPU time and accuracy.

The comparisons performed by Placzek et al. [36] reveal that for our application, the explicit algorithm adapted from [41] (referred to as the blended procedure in [36]) provides satisfactory results, as it combines a good accuracy and a relatively small CPU time. The method used for the time integration of Eq. (4) is based on an explicit algorithm, better than the one tested by Sigrist and Abouri [42]. Indeed, the numerical damping is dramatically reduced by combining a centered upwind and downwind discretization scheme for the prediction of the displacement. The steps of the coupling algorithm are the following:

1. Initialization for the first iteration $\left(x_{0}, v_{0}, a_{0}\right.$ and $F_{0}$ are the initial displacement, velocity, acceleration and force):

$y^{n}=x_{0}, \quad \dot{y}^{n}=v_{0}, \quad \ddot{y}^{n}=a_{0}, \quad F_{y}^{n}=F_{0}$

2. Explicit prediction of the cylinder acceleration for the time step $t_{n+1}$ using Eq. (4):

$\ddot{y}^{n+1}=\frac{F_{y}^{n}}{m}-\frac{k}{m} y^{n}$

3. Evaluation of the cylinder velocity and displacement with linear approximations ( $\delta t$ is the fluid time step, and $\theta$ is the blending factor):

$\dot{y}^{n+1}=\dot{y}^{n}+\delta t \ddot{y}^{n+1}$

$y^{n+1}=y^{n}+\delta t\left[(1-\theta) \dot{y}^{n}+\theta \dot{y}^{n+1}\right]$

4. Mesh update: computation of the new mesh configuration (according to the displacement $y^{n+1}$ evaluated in the preceding step) with the moving mesh procedure.

5. Resolution of the Navier-Stokes equations with the CFD code on the new mesh configuration to obtain $F_{y}^{n+1}$.

6. Return to step 2 for the next time step.

The previous procedure is implemented in a coupling subroutine handled by the CFD code at each time step before solving the flow field. This provides a numerical basis for more general code coupling procedures for future applications. Several numerical tests conducted by Sigrist [41] and Placzek et al. [36] have shown that $\theta=0.5$ pro- 
duces the smallest numerical damping in the coupled fluid/ structure calculation. Hence application of such algorithms to numerical simulations of VIV is straightforward; from a numerical point of view, this algorithm is not prone to alter the simulation of fluid-structure energy exchanges since the numerical damping is reduced.

Finally, it is worth mentioning that in both cases (forced and free oscillations), the cylinder motion is taken into account by the fluid model which is written in an arbitrary Lagrangian-Eulerian (ALE) formulation (see [10]). This formulation slightly modifies the Navier-Stokes equations by introducing an additional term related to the mesh motion: the convective term in Eq. (1) is changed in $\operatorname{div}[\mathbf{u} \otimes(\mathbf{u}-\mathbf{w})]$, where $\mathbf{w}$ is the mesh velocity field. Thanks to the moving mesh algorithm adapted from the one developed by Sigrist [41], the mesh velocity $\mathbf{w}$ is computed for each cell and the mesh can therefore be updated from the knowledge of the wall cylinder motion $y$. Combined with the fact that the size of the ring where the mesh moves can be easily chosen so that the mesh distortion remains small, this procedure which has already been used successfully by Sigrist [41] and Sigrist and Abouri [42] turns out to be also efficient in the case of an infinite domain.

\section{Fixed cylinder wake}

The wake of a fixed cylinder is investigated here for four Reynolds numbers in the permanent regime $(5 \lesssim R e \lesssim 49)$ and four others in the 2D periodic regime (49 $\lesssim e \lesssim 190$ ). The mesh remains stationary and several characteristic parameters of the wake are checked to control the validity and the accuracy of the numerical model.

\subsection{Permanent regime}

Simulations are carried out until the convergence residual becomes smaller than $10^{-7}$. The Reynolds numbers investigated are 10, 20, 30 and 40, all below the Hopf bifurcation between the permanent and the periodic regime, and the wake is thus characterized by two recirculation zones attached to the rear cylinder wall. They are recognizable by the strong vorticity $\zeta=\nabla \wedge \mathbf{u}$ and by low pressure levels, as it can be seen in Fig. 2.

The first parameter controlled is the length $L_{\mathrm{r}}$ of the recirculation zone which is defined by the downstream distance on the central line of the wake where the velocity is null. Values of $L_{\mathrm{r}}$ are easily obtained by plotting the velocity versus the distance after the cylinder wall for each Reynolds number (Fig. 3a). In the same way, values of the separation angle $\theta_{\mathrm{s}}$ are obtained by plotting the vorticity at the cylinder wall against the angular position: $\theta_{\mathrm{s}}$ corresponds to the angle where the vorticity becomes null (Fig. 3b). The evolution of these two parameters is presented in Figs. $4 a$ and $b$. The recirculation length is a linear function of the Reynolds number and a least squares approximation provides the following expression: $L_{\mathrm{r}}=0.0671 R e-0.4155$. The intersection
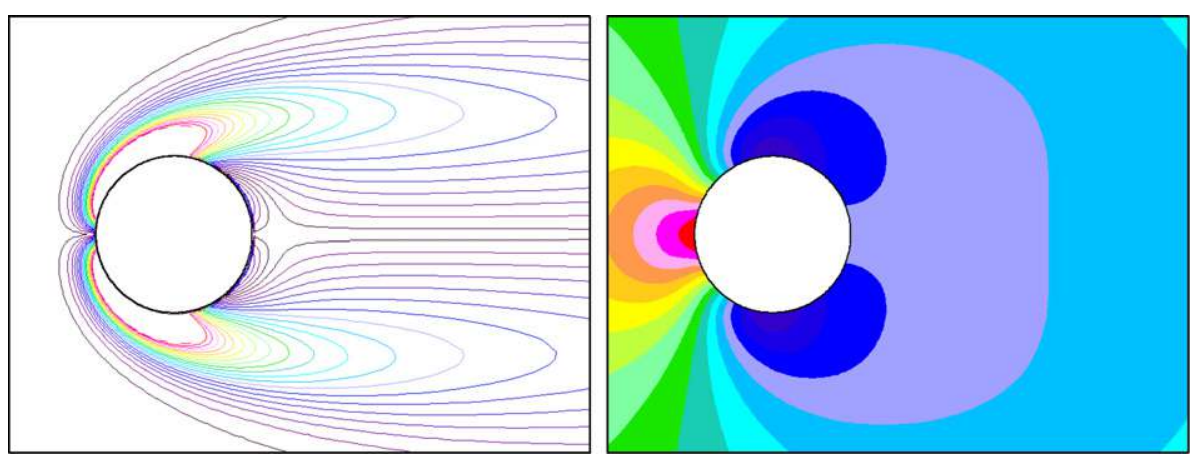

Fig. 2. Details of the vorticity contours (left) and pressure field (right) in the wake at $R e=40$.

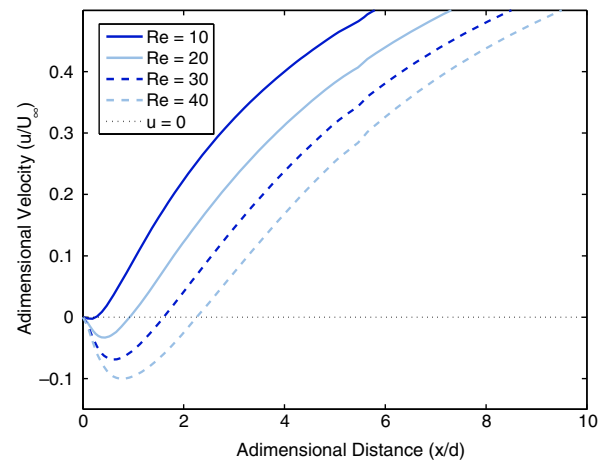

(a) Streamwise velocity versus the downstream distance after the cylinder wall

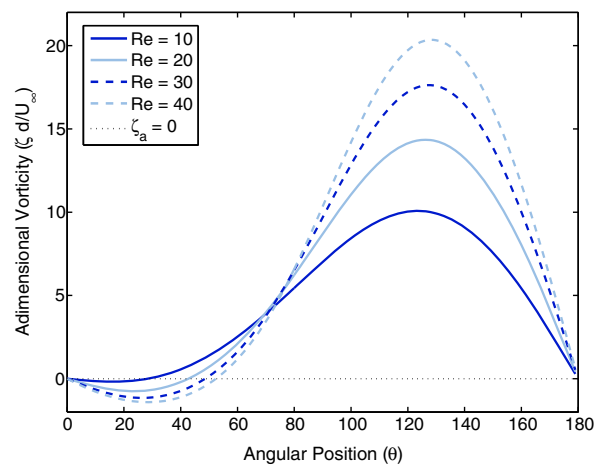

(b) Vorticity at the cylinder wall versus the angular position on the cylinder wall

Fig. 3. Plots used for the determination of the recirculation length $L_{\mathrm{r}}$ and the separation angle $\theta_{\mathrm{s}}$. 


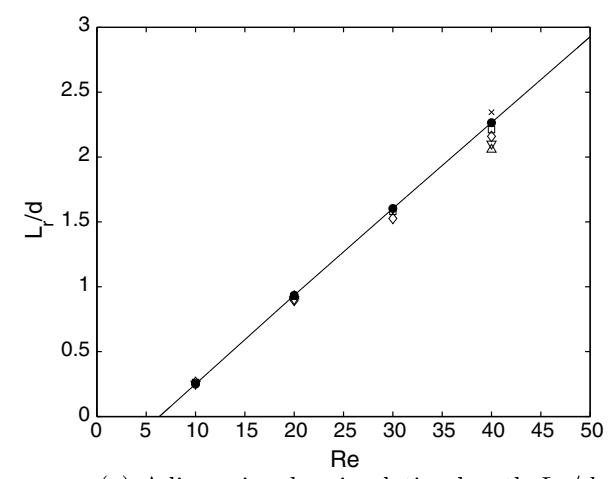

(a) Adimensional recirculation length $L_{r} / d$

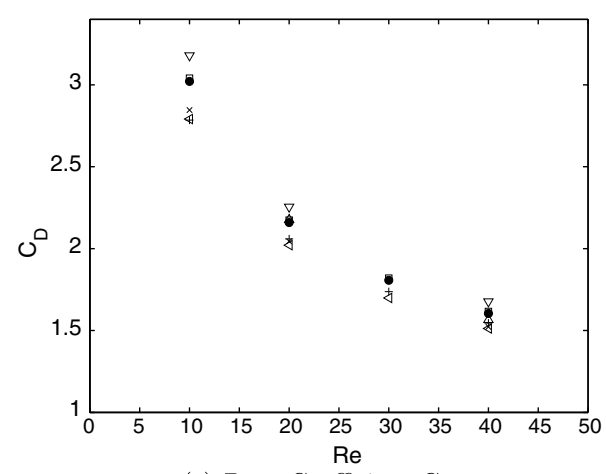

(c) Drag Coefficient $C_{D}$

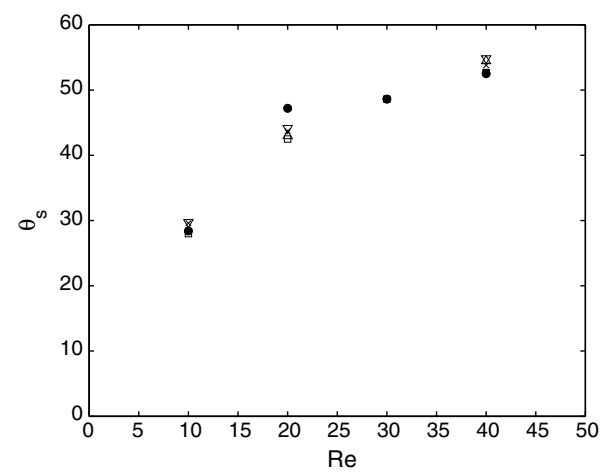

(b) Separation angle $\theta_{s}\left({ }^{\circ}\right)$

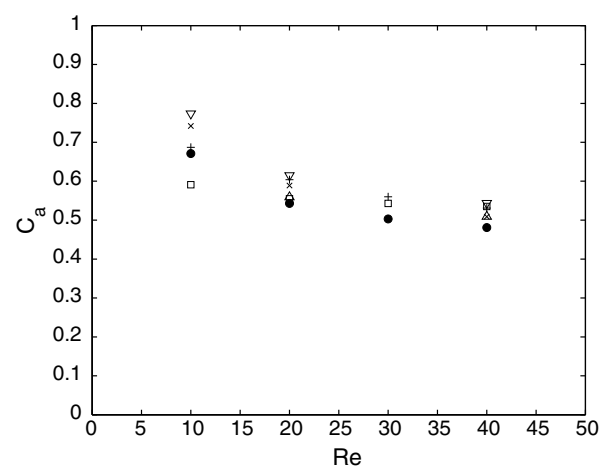

(d) Suction Coefficient $C_{a}$

Fig. 4. Comparison of the geometric wake characteristics and aerodynamic force coefficients for the permanent regime $(\operatorname{Re}<49)$. $(\bullet)$ present simulations,

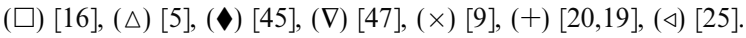

of this line with the axis of abscissa defines the value of the critical Reynolds number $R e_{\mathrm{c}}$ from which the recirculation zones appear. The value found here $R e_{\mathrm{c}}=6.19$ is slightly surevaluated compared to the one proposed by Gerouache [16] $\left(R e_{\mathrm{c}}=5.74\right)$, and Socolescu [45] $\left(R e_{\mathrm{c}}=5.84\right)$. Values of the recirculation length are close to the common values obtained in other studies but the discrepancy between the results increases with $R e$. For the separation angle $\theta_{\mathrm{s}}$, the values are also in good agreement with other studies. For these two parameters, the relative errors (computed as the absolute value of the difference between the value obtained with the present simulation and the average of the values found in other studies divided by this same value) remain inferior to $5 \%$ for the four Reynolds numbers tested.

Values of the drag $C_{\mathrm{D}}$ and suction coefficients $C_{\mathrm{a}}$ are also controlled. As expected, the lift coefficient $C_{\mathrm{L}}$ remains null because of the perfect symmetry of the flow field. The expressions of the aerodynamic coefficients are reminded below:

$C_{\mathrm{D}}=\frac{F_{\mathrm{D}}}{1 / 2 \rho U_{\infty}^{2} d} \quad C_{\mathrm{L}}=\frac{F_{\mathrm{L}}}{1 / 2 \rho U_{\infty}^{2} d} \quad C_{\mathrm{a}}=\frac{p_{\infty}-p_{0}}{1 / 2 \rho U_{\infty}^{2}}$

$F_{\mathrm{D}}$ (resp. $F_{\mathrm{L}}$ ) is the drag (resp. the lift) force by length unit, $p_{\infty}$ is the reference pressure and $p_{0}$ is the pressure at the rear stagnation point. Values of the drag coefficient (see Fig. 4c) are close to those of Gerouache [16] and Tuann and Olson [47] who also used a small aspect ratio, but as mentioned before, they are slightly surestimated compared to other studies like those of Lange et al. [25], Henderson [20], Dennis and Chang [9] where the aspect ratio $H / D$ is greater. However, the agreement becomes better when Re increases and the relative errors on the drag coefficient remain inferior to $5 \%$ for each Reynolds number. Finally, the errors on the suction coefficient $C_{\mathrm{a}}$ are slightly larger but still under $10 \%$. This can be explained by the fact that $C_{\mathrm{a}}$ is a local variable which is, from the computational point of view, very sensitive to numerical error.

\subsection{Periodic vortex shedding regime}

Transient simulations are now investigated. The simulation time is chosen long enough to observe about 15 vortices shedding once the periodic regime is established. The time step is set to about $1 / 150$ of the Strouhal period during the transient phase at the beginning of the computations. Once the periodic regime is reached, the time step is then divided by 4 to increase the accuracy of the results. The CPU time stand at about $26 \mathrm{~h}$. To obtain the Von Kármán streets, the symmetry of the wake has to be numerically broken thanks to a numerical artefact: $1 \%$ amplitude arbitrary noise is added to the incident velocity for that purpose. This perturbation is only maintained during a small time interval.

The Reynolds numbers investigated now are 60, 80, 100 and 120. In this case, the wake is composed of two staggered rows of vortices being shed alternately from either 


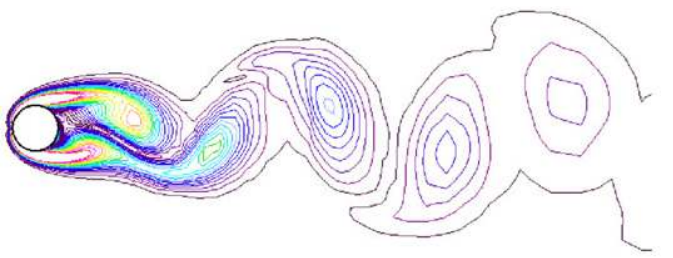

(a) $t=t_{0}$

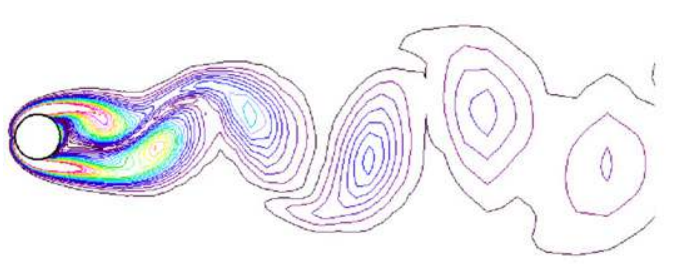

(c) $t=t_{0}+2 / 3 T_{S}$

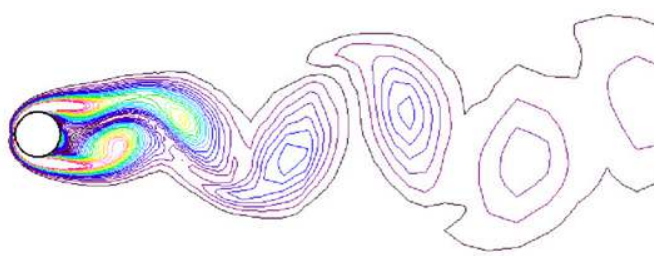

(b) $t=t_{0}+1 / 3 T_{S}$

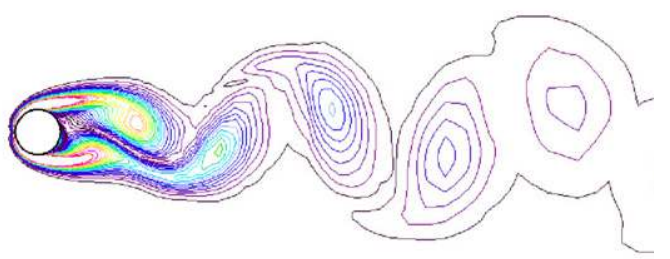

(d) $t=t_{0}+T_{S}$

Fig. 5. Vorticity contours in the wake over one Strouhal period at $R e=100$.

side of the cylinder. Fig. 5 shows the vorticity contours in the wake of the cylinder over a complete Strouhal period $T_{\mathrm{S}}$ at $R e=100$. At $t=t_{0}$, a vortex is forming in the lower side of the wake and is then completely detached from the cylinder wall at $t=t_{0}+1 / 3 T_{\mathrm{S}}$. On the following snapshot, the vortex of the upper side is about to be inserted between the lower vortex formed previously and a new vortex which is forming. At $t=t_{0}+T_{\mathrm{S}}$, the vortex in the upper side is completely detached and the wake topology is exactly the same as the one observed at $t=t_{0}$.

The periodicity of the shedding leads naturally to the fluctuation of the aerodynamic coefficients which will be denoted now by $C_{\mathrm{D}}^{\prime}, C_{\mathrm{L}}^{\prime}$ and $C_{\mathrm{a}}^{\prime}$ for the fluctuating values and by $\overline{C_{\mathrm{D}}^{\prime}}, \overline{C_{\mathrm{L}}^{\prime}}$ and $\overline{C_{\mathrm{a}}^{\prime}}$ for the mean values. These latter are evaluated as the average value of the fluctuating coefficients over several periods chosen after the transient. The convergence of the coefficients is shown in Fig. 6 at $R e=100$. The first part of the time series $\left(t^{*}<10\right)$ exhibits the transient phase during which the perturbation initially introduced arrives on the cylinder and causes the shedding. The periodic state reached is characterized by the oscillation of the drag coefficient at twice the lift frequency. The fluctuations of the suction coefficient are governed by two frequencies: the main frequency is equal to the lift oscillation frequency and the secondary is identical to the drag oscillation frequency.

The Strouhal frequency $f_{\mathrm{S}}$ can be defined as the lift coefficient frequency or the fluctuation frequency of velocity for any point in the near wake. The two frequencies are equal and the Strouhal number is obtained by the following relation:

$S t=f_{\mathrm{S}} \frac{d}{U_{\infty}}$

The values of the Strouhal numbers obtained here are compared in Fig. 7a to the analytical expressions of Fey et al. [14], Norberg [30], Roshko [38], Williamson and Roshko $[49]$ and to other values from $[16,25,20]$. Although the evolution of the Strouhal is well respected, the values computed are slightly surestimated; however the relative error does not rise above $3.5 \%$. This should one more time be related to the small aspect ratio used here which could also explain the great values of the mean drag coefficient $\overline{C_{\mathrm{D}}^{\prime}}$ compared to those of [20] (see Fig. 7b). The mean value of the lift $\overline{C_{\mathrm{L}}^{\prime}}$ is null and we therefore use the maximal value reached $C_{\mathrm{L}, \text { max }}^{\prime}$ or the root mean square value $C_{\mathrm{L}, \mathrm{rms}}^{\prime}$ as rep-
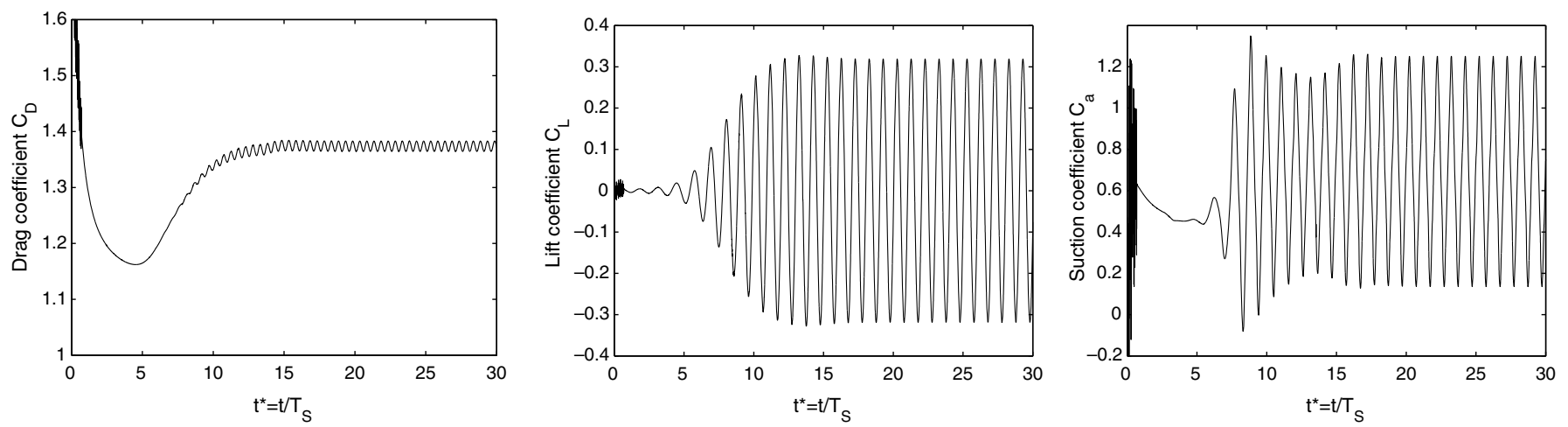

Fig. 6. Convergence of the aerodynamic fluctuating coefficients at $R e=100$. From left to right: drag coefficient $C_{\mathrm{D}}^{\prime}$, lift coefficient $C_{\mathrm{L}}^{\prime}$ and suction coefficient $C_{\mathrm{a}}^{\prime}$. 


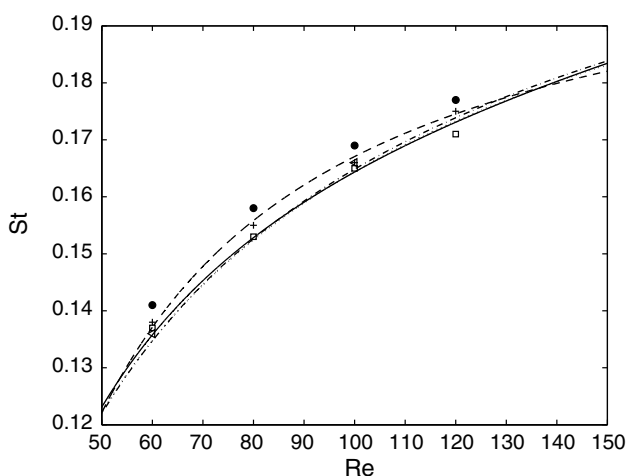

(a) Strouhal Number $S t$

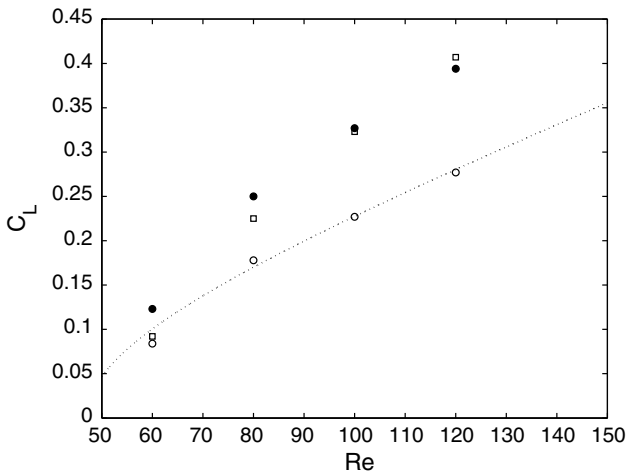

(c) Maximal and rms Lift Coefficient $C_{L, \max }^{\prime}$ and $C_{L, \mathrm{rms}}^{\prime}$

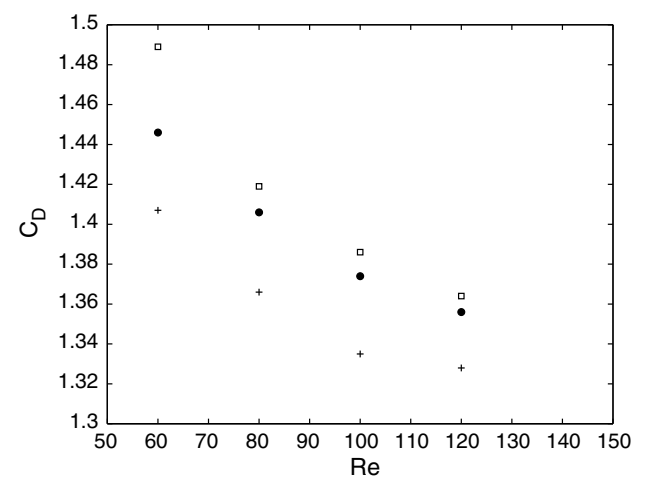

(b) Mean Drag Coefficient $\overline{C_{D}^{\prime}}$

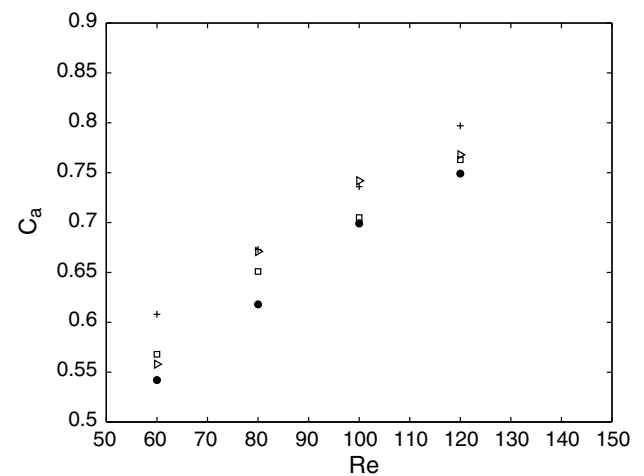

(d) Suction Coefficient $\overline{C_{a}^{\prime}}$

Fig. 7. Comparison of the geometric wake characteristics and aerodynamic force coefficients for the periodic regime $(49<R e<190)$. $(\bullet)$ present simulations, $(\square)[16],(+)[20,19],(\triangleleft)$ [25], (-) and ( $)$ [48], (----) [38], (-) [14]. For the lift coefficient, the maximal values of the present simulations $(\bullet)$ are compared to the results of $[16](\square)$; the rms values are represented on the same graph with empty circles $(\bigcirc)$ and compared to the analytical expression of $[30](\cdots)$.

resentative parameters. The graph Fig. 7c presents the evolutions of the maximal lift coefficient $C_{\mathrm{L}, \max }^{\prime}$ compared to the values of Gerouache [16] and of the rms lift coefficient $C_{\mathrm{L}, \mathrm{rms}}^{\prime}$. This latter is compared to the empirical expression derived by Norberg [30] and show a very good agreement. Finally the mean suction coefficient $\overline{C_{\mathrm{a}}^{\prime}}$ is compared to the results of $[16,48,20]$ in Fig. 7 d: the $\overline{C_{\mathrm{a}}^{\prime}}$ is slightly underestimated but the trend is respected. The accuracy of the results is rather satisfactory, especially concerning the values of the Strouhal number and of the lift coefficient which should be precise as they will play a major role in the following study of the VIV.

\section{Forced oscillations}

Simulations are now performed for a cylinder forced to oscillate at the frequency $f_{0}$ which is better described by the frequency ratio $F=f_{0} / f_{\mathrm{S}}$. Computations are run for a Reynolds number constant and equal to 100 from the solution for the fixed cylinder, i.e. when the Von Kármán streets are already present. Only the forced frequency $f_{0}$ and possibly the amplitude $A$ are changed. The lock-in zone is defined by the domain where the vortex shedding frequency diverges from the value expected at the Reynolds number considered and locks on the frequency of the forced oscillations: this zone is represented in the plane $(A, F)$ in
Fig. 8 according to the limits established by Koopmann [24]. The lock-in zone is comprised between two limits almost symmetrical with respect to the axis $F=1.0$, although slight differences confirmed by the numerical simulations of Anagnostopoulos [3] are observed for low amplitudes. In order to highlight the different response regimes of the cylinder, the amplitude $A$ is kept constant

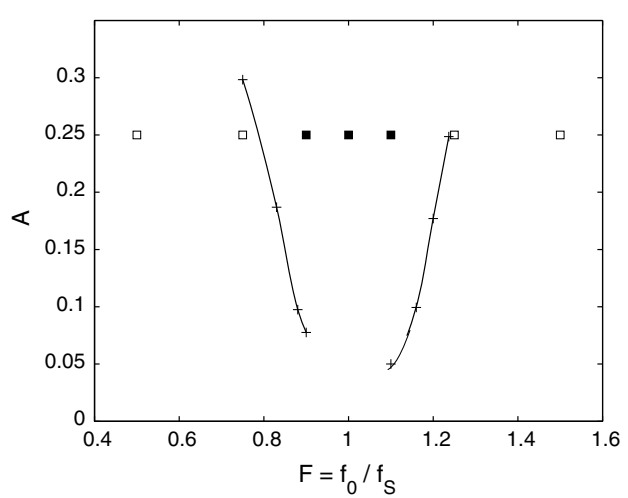

Fig. 8. Representation of the lock-in zone in the plane $(A, F)$ for forced transverse oscillations. The solid lines represent the frontiers of the lock-in zone according to the data points (+) of Koopmann [24]. The simulations performed here are represented with squares: filled squares $(\boldsymbol{\square})$ correspond to locked configurations whereas empty squares $(\square)$ indicate unlocked ones. 
beyond a certain level and $F$ varies over a range wide enough so that the lock-in zone should be crossed.

\subsection{Cylinder response and lock-in zone}

The cylinder response is studied for several frequency ratios $F$ between 0.50 and 1.50 while the amplitude $A$ is kept constant and equal to 0.25 . We present in the following two types of responses, the first in the lock-in zone and the second out of it. For $A=0.25$, the upper and lower limits are approximately located at $F=0.75$ and $F=1.25$ according to the frontiers established by Koopmann [24].

\subsection{Locked configurations}

Two cases are presented here to illustrate the locked configurations: $F=0.90$ and $F=1.10$. Time series of the aerodynamic coefficients are characterized by a pure sinusoidal response (see Fig. 9, col. 1) and exhibit a strong increase of the drag coefficient: indeed, for the fixed cylinder the mean drag coefficient was $\overline{C_{\mathrm{D}}^{\prime}}=1.37$ (see Fig. 7b), whereas the values reach now $\overline{C_{\mathrm{D}}^{\prime}}=1.50$ for $F=0.90$ and $\overline{C_{\mathrm{D}}^{\prime}}=1.75$ for $F=1.10$. The maximal value of the lift coefficient $\left(C_{\mathrm{L}, \max }^{\prime}=0.33\right.$ for the fixed cylinder $)$ is smaller for $F=0.90\left(C_{\mathrm{L}, \max }^{\prime}=0.28\right)$ but increases then when $F=1.10$ $\left(C_{\mathrm{L}, \max }^{\prime}=1.44\right)$. The spectra of the lift coefficient presented in Fig. 9, col. 2 highlight this sinusoidal response and clearly show that the main frequency is $f_{0}$ since $f^{*}=f / f_{0} \approx 1.0$. This indicates that the aerodynamic forces are now governed by the forced frequency instead of the Strouhal frequency $f_{\mathrm{S}}$ determined above for a fixed cylinder. In the same way, PSDs of the drag coefficient - not represented here - would show a main peak at $f^{*} \approx 2.0$, meaning that the forced frequency also controls the drag fluctuations. This periodicity can also be observed in the wake: visualizations of the vorticity contours in the wake (not shown here) would be exactly the same at two instants separated by one period $T_{0}=1 / f_{0}$.

The phase portraits of the system are also a very practical tool to analyze the response. Indeed, they represent the energy transfer (product of the fluctuating lift force characterized by the fluctuating lift coefficient $C_{\mathrm{L}}^{\prime}$ and the adimensional cylinder displacement $y^{*}=y(t) / d$ ) between the motion of the cylinder and the fluid and thus provide an interesting description of how the system behaves. Phase portraits for the two cases under study are therefore given in Fig. 9, col. 3 as a complement to the PSDs. The existence of a unique limit cycle is the result of the perfect undamped sinusoidal response and the inclination of the cycle gives an estimation of the phase angle between the imposed displacement and the lift.

\subsection{Unlocked configurations}

The lock-in region is defined like Nobari and Naredan [29] as the domain where the evolution of $C_{\mathrm{L}}^{\prime}$ is purely sinu-
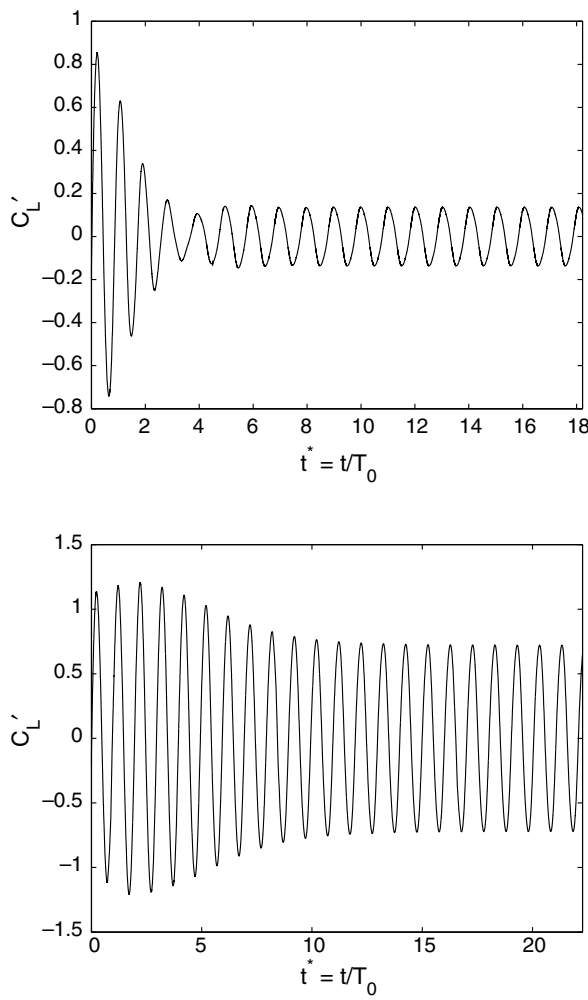

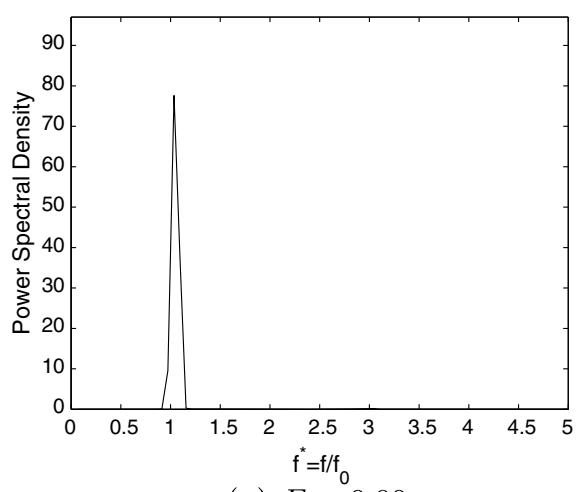

(a) $F=0.90$

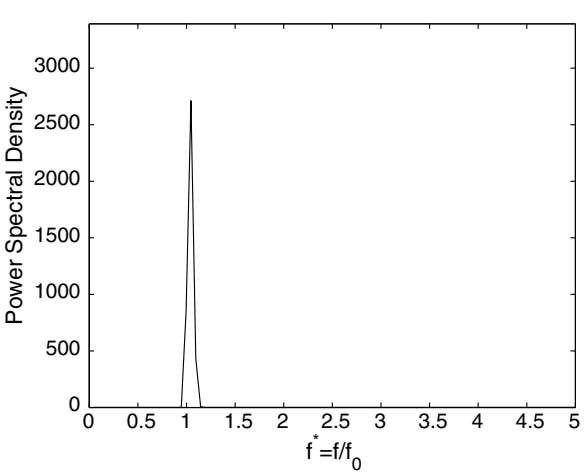

(b) $F=1.10$
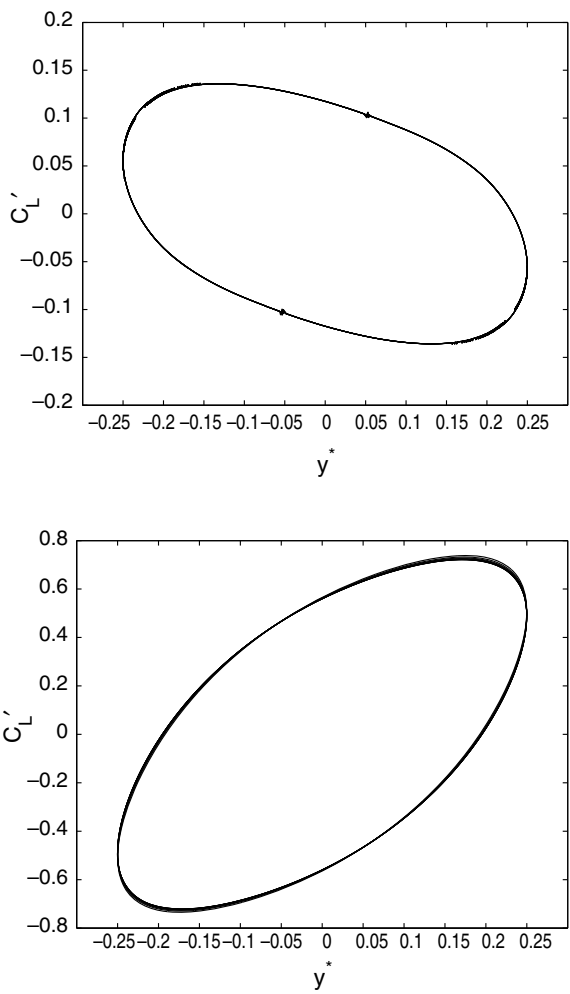

Fig. 9. Time series of the fluctuating lift coefficient $C_{\mathrm{L}}^{\prime}$ (first column), PSD of the fluctuating lift coefficient (second column) and phase portraits (third column) for the cylinder forced to oscillate at $F=0.90$ and $F=1.10$. 
soidal and governed by the forced oscillation frequency. This means that only one peak at $f^{*}=1.0$ should be present in the frequency spectrum. A locked or unlocked wake is therefore easily identified not only thanks to the PSDs, but also and preferably with the analysis of the phase portraits.

The frequency ratios $F=0.50$ and $F=1.50$ are chosen to illustrate this as they lead to an unlocked wake. Time series of the lift coefficient are no longer purely sinusoidal and a beating behavior is observed: the signal is not periodic over two successive cycles of oscillation but over several ones. The time series are therefore characterized by a cycle-to-cycle period, which is different from the "real" period, this latter being defined by the time interval after which the signal is exactly the same. The beating behavior has also been observed numerically by Anagnostopoulos [3] who noted that when the frequency ratio $F$ was greater than 1 or smaller than 1 and outside the lock-in zone, the flow was not absolutely periodic at subsequent cycles but a quasi-periodic flow pattern occurred. The presence of more than one peak in the frequency spectra has also been observed by Nobari and Naredan [29] or Mittal and Kumar [27] for transverse or in-line oscillations. For $F=0.50$ (Fig. 10a, col. 1), the cycle-to-cycle oscillation is associated to the Strouhal period $T_{\mathrm{S}}$ but it is obvious that the signal is not $T_{\mathrm{S}}$ periodic. The beating period $T_{\mathrm{B}}$ describing the periodicity of the signal is in this case equal to the forced oscillation period $T_{0}$ and by definition of $F$ we have $T_{\mathrm{S}}=F T_{0}=0.5 T_{0}$. The case $F=1.50$ (Fig. $10 \mathrm{~b}$, col. 1 ) is more complex: this time, the cycle-to-cycle oscillation is associated to the forced oscillation period $T_{0}$ and the Strouhal period is $T_{\mathrm{S}}=1.5 T_{0}$. The beating period $T_{\mathrm{B}}$ is now equal to $8 T_{0}$.

The PSDs presented in Fig. 10, col. 2 for the cases $F=0.50$ and $F=1.50$ exhibit now two peaks. The first peak at $f^{*}=1.0$ corresponds to the forced oscillation frequency $f_{0}$ and is still present on the two spectra but is alternately the main peak $(F=1.50)$ or the secondary one $(F=0.50)$. The second peak comes from the Strouhal frequency $f_{\mathrm{S}}$ evaluated for the fixed cylinder and is therefore located at $f^{*} \approx f_{\mathrm{S}} / f_{0}=1 / F=2$ for $F=0.50$ and $f^{*} \approx 2 / 3$ for $F=1.50$. The unlocked configurations are thus characterized by the presence of the two frequencies $f_{0}$ and $f_{\mathrm{S}}$, each one playing now a role in the vortex shedding process. The main peak is always responsible for the cycle-to-cycle oscillation whereas the low-frequency peak affects the periodicity of the signal. For the case $F=0.50$, the beating is enough energetic and is therefore identified on the spectra as the low-frequency peak at $f^{*}=1.0$ (Fig. 10a, col. 2): the beating frequency $f_{\mathrm{B}}$ giving the periodicity of the time series of the $C_{\mathrm{L}}^{\prime}$ is thus approximately equal to $f_{0}$. For the case $F=1.50$, the second peak at $f^{*}=2 / 3$ corresponds to the Strouhal frequency $f_{\mathrm{S}}$. In this case, the beating is less energetic in the sense that the difference of amplitude
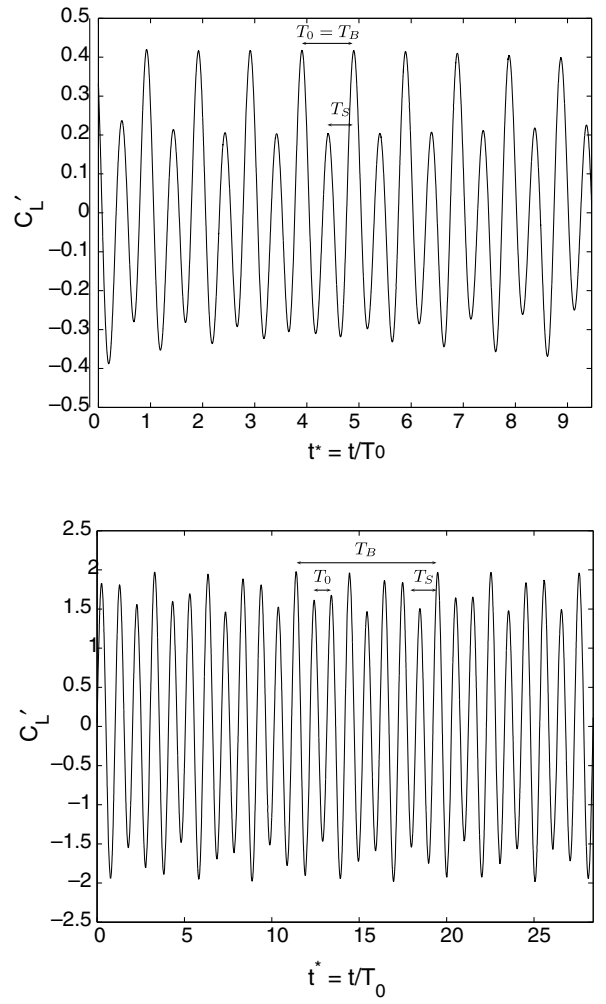

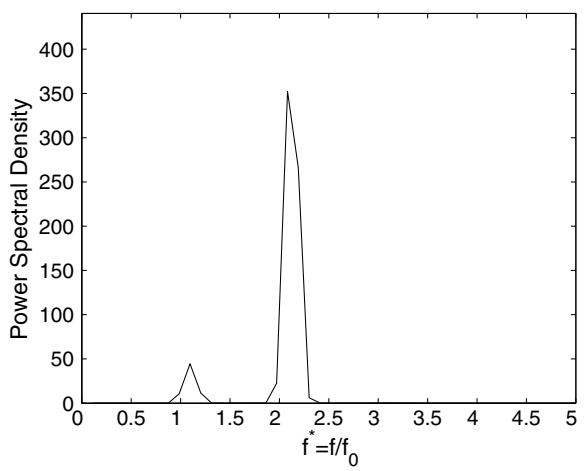

(a) $F=0.50$

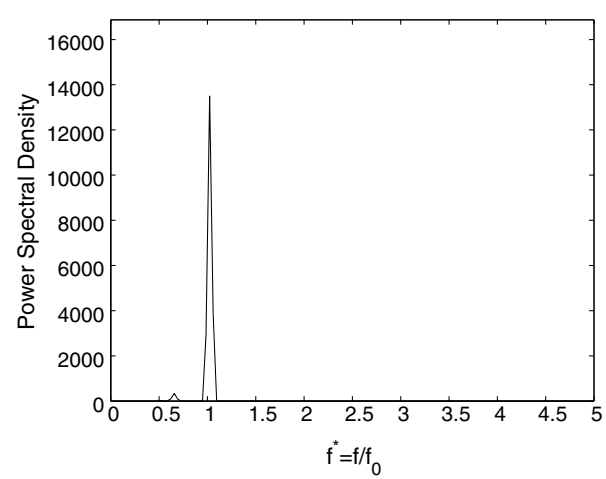

(b) $F=1.50$
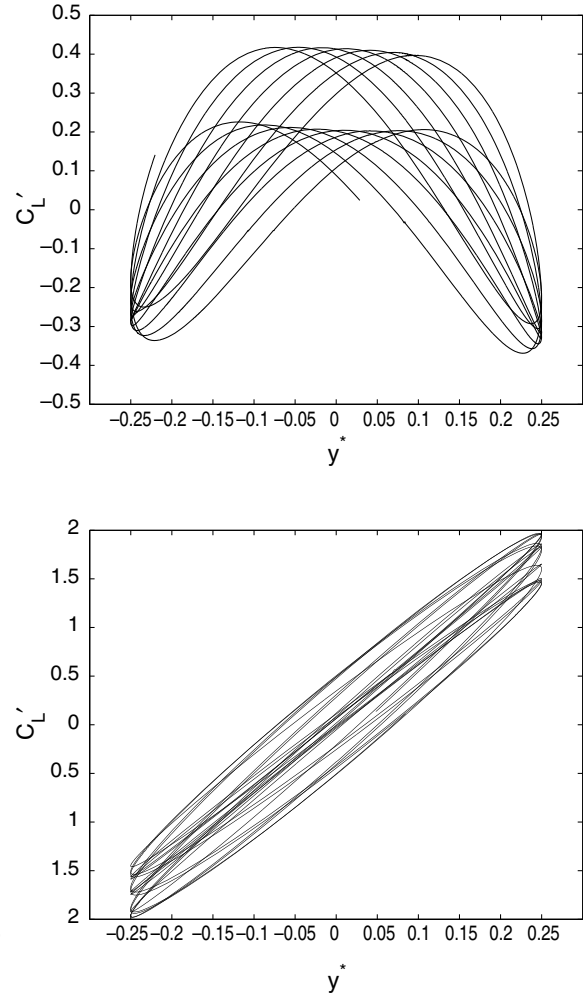

Fig. 10. Time series of the fluctuating lift coefficient $C_{\mathrm{L}}^{\prime}$ (first column), PSD of the fluctuating lift coefficient (second column) and phase portraits (third column) for the cylinder forced to oscillate at $F=0.50$ and $F=1.50$. 
between successive cycles is smaller (for $F=0.50$, the difference of amplitude was about $50 \%$ whereas now it is less than $25 \%$ ). Combined with the fact that the time simulation is possibly not long enough, the beating frequency is not detected by the spectral analysis represented in Fig. 10b, col. 2.

Finally, the phase portraits (Fig. 10, col. 3) are dramatically different from those obtained earlier. Indeed, they highlight the presence of more than one frequency in the signal which causes a fluctuation of the $C_{\mathrm{L}}^{\prime}$ value between two successive cycles. These fluctuations are characterized by a different path between two cycles and as a result, there are many ways in the interior of the limit cycle.

Although the beating frequency $f_{\mathrm{B}}$ is sometimes hardly visible on the spectra (cf. the case $F=1.50$ ), it plays an important role for the vortex shedding process. Even if it is not visible on the spectra, the beating period $T_{\mathrm{B}}=1 / f_{\mathrm{B}}$ can be evaluated by plotting the vorticity contours at different instants: the time interval between two identical snapshots provides an estimation of $T_{\mathrm{B}}$. Fig. 11 represents the vorticity contours at $t=t_{0}, t=t_{0}+T_{0}, t=t_{0}+T_{\mathrm{S}}$, and $t=t_{0}+T_{\mathrm{B}}$. In the present case, we find $T_{\mathrm{B}}=8 T_{0}$. This value agrees with the time series of the $C_{\mathrm{L}}^{\prime}$ (Fig. 10b, col. 1) where it can be seen that the amplitude at two instants separated by $T_{\mathrm{B}}$ is the same. It is clear from the vorticity contours that the periodicity of the wake is now governed by the beating period $T_{\mathrm{B}}$ (the first and last pictures are exactly the same) instead of the Strouhal period $T_{\mathrm{S}}$ or the forced oscillation period $T_{0}$ as it could be expected by seeing the PSD. Inside the lock-in zone, we observed that the wake was governed by the period $T_{0}$ of the forced oscilla- tion which was also the period of lift coefficient fluctuations. On the contrary, the behavior is more complex outside the lock-in zone: according to the relative importance of the peaks in the PSD (Fig. 10), it can be argued that the cycle-to-cycle period of the lift is governed by the Strouhal frequency below the lower limit of the lockin zone (resp. the forced oscillation frequency upon the upper limit). However, the periodicity of the $C_{\mathrm{L}}^{\prime}$ is associated to a certain beating period $T_{\mathrm{B}}$ which is a multiple of the cycle-to-cycle period and which corresponds to the periodicity of the vortex shedding process.

\subsection{Aerodynamic coefficients}

In addition to a shift of the shedding frequency, the lock-in region is also characterized by an increase of the aerodynamic coefficients, when compared to the fixed cylinder case. Fig. 12 represents the evolution of the mean drag and maximal lift coefficient when the frequency ratio $F$ is increased and the amplitude $A=0.25$ is constant. The evolution of the mean suction coefficient is not given here as it is quite similar to the mean drag coefficient, the maximal value being obtained in both cases at $F=1.10$. The aerodynamic coefficients are practically always greater than the fixed cylinder values which are represented with dashed lines. The shape of the drag curve Fig. 12a is characterized by a maximum inside the lock-in zone and the drag coefficient seems to relapse near the fixed cylinder value outside of the lock-in zone. On the contrary, after a small drop, the lift is increasing from the beginning of the lock-in zone. Outside the lock-in zone, this amplification seems to

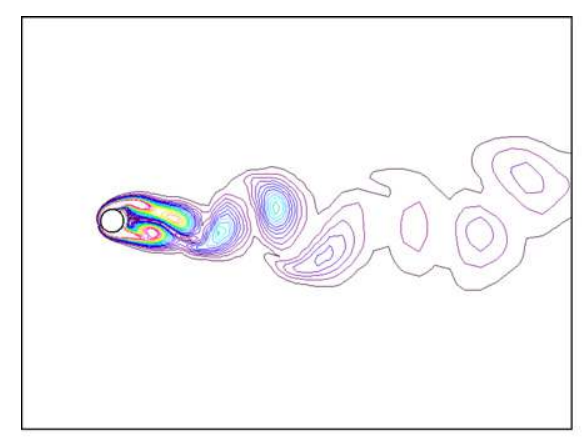

(a) $t=t_{0}$

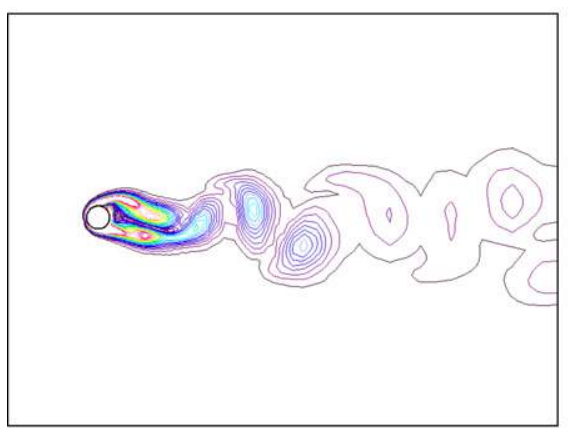

(c) $t=t_{0}+T_{S}$

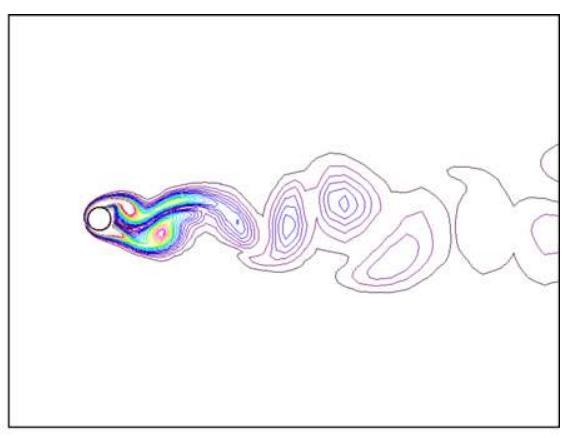

(b) $t=t_{0}+T_{0}$

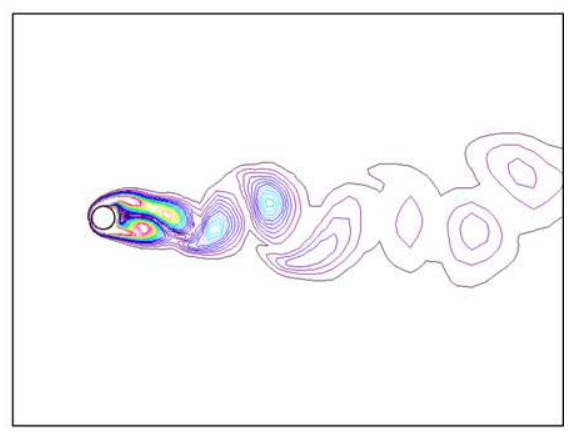

(d) $t=t_{0}+T_{B}$

Fig. 11. Vorticity contours at different strategic moments for $F=1.50$. 

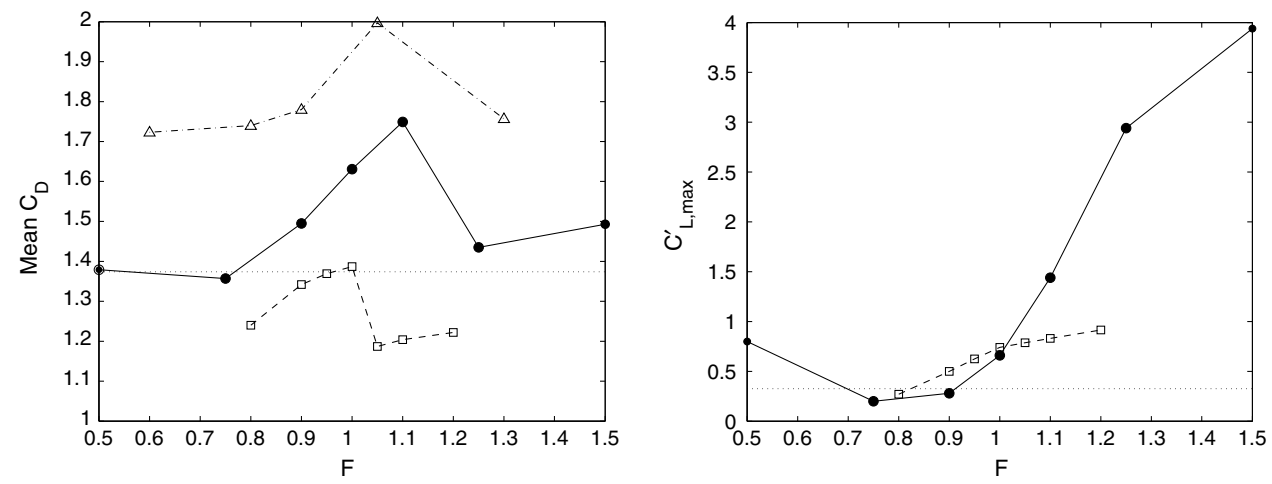

Fig. 12. Evolution of the mean drag and maximal lift coefficients with the frequency ratio $F$. The dashed lines give the values of the mean drag $\overline{C_{\mathrm{D}}^{\prime}}$ and of the maximum lift coefficient $C_{\mathrm{L}, \max }^{\prime}$ for the fixed cylinder case. The results are compared to those of Anagnostopoulos [3] ( $\square$ ) and Nobari and Naredan [29] $(\triangle)$.

become less pronounced when $F$ is further increased. The computed curves are compared to the results of Anagnostopoulos [3] and Nobari and Naredan [29]. The shape of the drag curve is globally similar with a maximum in the lock-in zone but the discrepancies between the values are important. Although there are some small differences concerning the parameters of the model ( $R e=106$ for Anagnostopoulos [3], $A=0.2$ for Nobari and Naredan [29]), the discrepancies stem certainly from the values obtained for the fixed cylinder case: Anagnostopoulos found $\overline{C_{\mathrm{D}}^{\prime}}=1.28$, Nobari $\overline{C_{\mathrm{D}}^{\prime}}=1.72$ and the present study yields $\overline{C_{\mathrm{D}}^{\prime}}=1.37$. This explains that the drag curve is comprised between the two others, and recalling that the mean drag is slightly surestimated, one could expect that the curve would be shifted towards lower values if a greater aspect ratio had been used. Concerning the lift coefficient, the fixed cylinder case provided $C_{\mathrm{L}, \max }^{\prime}=0.33$ whereas Anagnostopoulos found $C_{\mathrm{L}, \max }^{\prime}=0.17$. There is a factor 2 between the two values and therefore the discrepancy between the curves Fig. 12b is very important. Despite the great differences, the two curves exhibit an increase of the lift coefficient inside the lock-in zone and a stabilization of the amplification near the end of the lock-in zone.

To conclude this paragraph, it is worth mentioning that a jump in the phase angle could be observed at the beginning of the lock-in zone. Carberry et al. [7] find experimentally at about $F=0.80$ a jump of the lift coefficient and of the phase angle $\phi$ defined as the shift between the cylinder displacement and the fluctuating lift force. Below this value, $\phi$ is approximately equal to $180^{\circ}$ and falls to $0^{\circ}$ at the critical value of $F$. The authors attribute this jump to a modification of the vortex shedding mode which appears in the wake. In the present study, it is also observed that the lift coefficient increases when $F$ increases but Carberry et al. [7] found out that the steepest slope is at $F=0.80$, whereas in the present case this happens later, between $F=1.00$ and 1.25 . The phase angle has only been evaluated in the lock-in zone where the fluctuations are purely sinusoidal. The present simulations show a smooth decrease of $\phi$ with $F$ and no real jump has been observed: the values found here are $\phi=113.8$ for $F=0.90, \phi=80.5$ for
$F=1.00$ and $\phi=47.7$ for $F=1.10$. A plausible reason is the difference in the Reynolds number between our study $(R e=100)$ and the one of Carberry et al. [7] where $R e=2300$. Although it is not yet clear, it has been noticed by Khalak and Williamson [22], Anagnostopoulos [3], Nobari and Naredan [29] that the change of vortex shedding mode is most of the time not observed at low Reynolds numbers, whereas it can be seen at higher Reynolds numbers [48].

\subsection{Vortex shedding modes}

Attention has also been paid here to the topology of the wake. The beating phenomenon described previously also appears at $(A, F)=(0.25,1.25)$. It has been analyzed and associated to a vortex merging mechanism for this couple of parameters by Placzek et al. [35]. Then greater values for the amplitude $A$ have been used to observe different shedding modes, which are commonly called the $2 \mathrm{P}$ and $\mathrm{P}+\mathrm{S}$ modes according to the appellations employed by Williamson and Roshko [49]. The values used to investigate other shedding modes are deduced from the vortex shedding map proposed by Williamson and Roshko [49].

First, the couple $(A, F)=(1.00,0.90)$ is chosen in the middle of the $2 \mathrm{P}$ shedding mode domain. The wake looks like a $2 \mathrm{~S}$ shedding mode but the vortices are stretched in the vertical direction because of the increased amplitude of oscillation (see Fig. 14a). When the wake structure is observed in detail, it is possible to discern a secondary vortex denoted $V_{2}$, whose intensity is very small. The vortices are still alternately shed from the upper and lower side of the cylinder as in a $2 \mathrm{~S}$ mode, but as they move downstream, the weakly energetic vortex $V_{2}$ slips over its following neighbor $V_{3}$ and forms the tail of its preceding neighbor $V_{1}$. The vortices are then assembled by asymmetric pairs $\left(V_{2}-V_{3}\right)$. Over one cycle period, two pairs are shed, so the vortex shedding mode looks like a $2 \mathrm{P}$ mode in which the vortices would not be identical in a same pair. As the small vortex is weakly energetic, it quickly disappears in the far wake. The vortex shedding mode in the far wake looks therefore like a $2 \mathrm{~S}$ mode with deformed vortices. It 

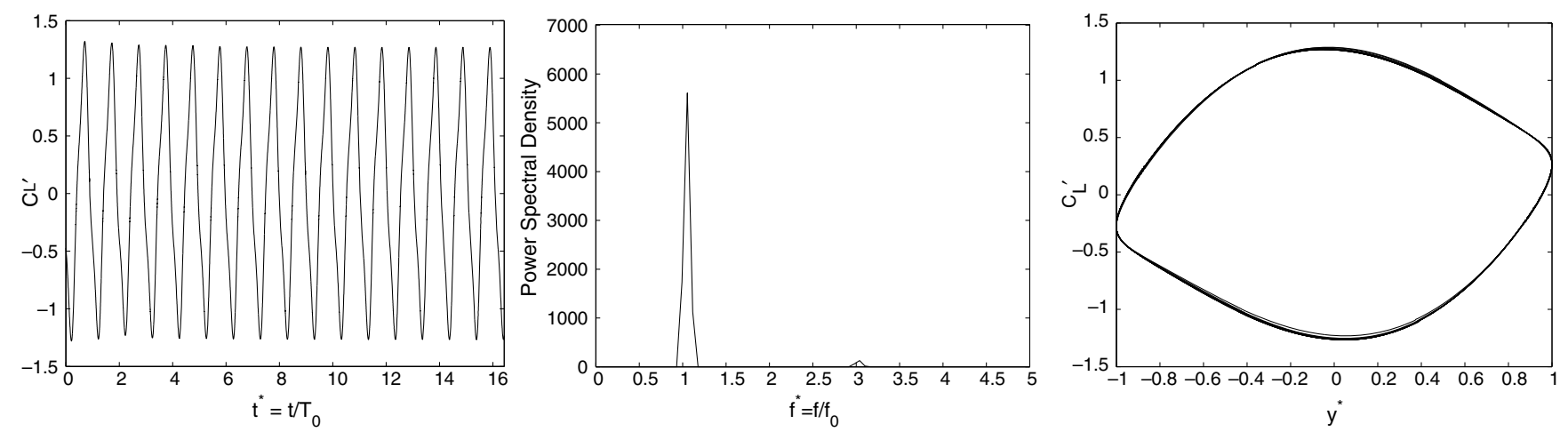

(a) $(A, F)=(1.00,0.90)$
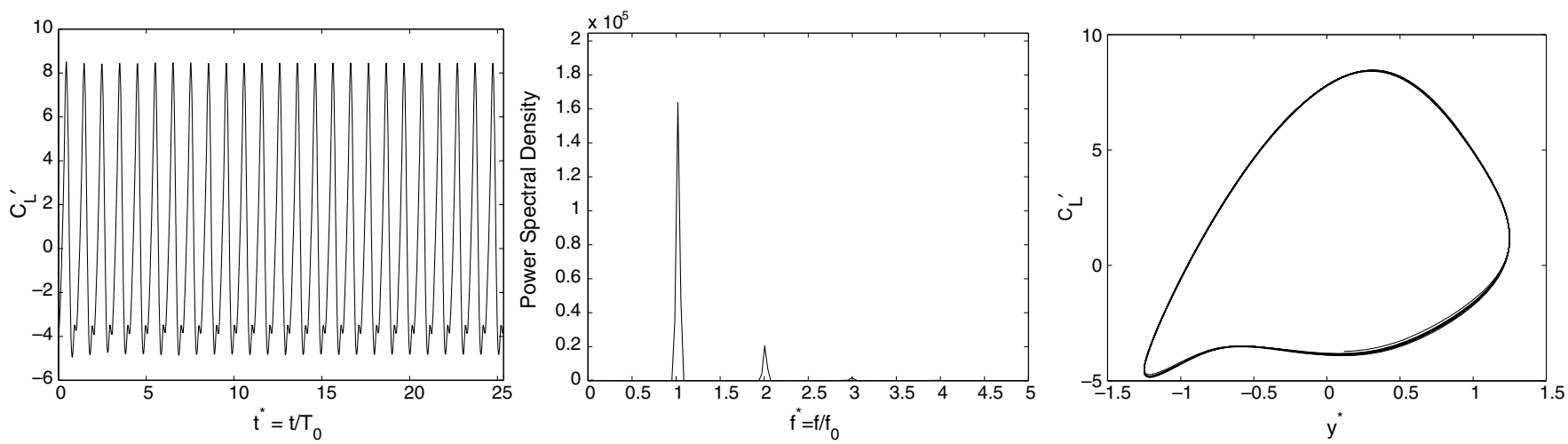

(b) $(A, F)=(1.25,1.50)$

Fig. 13. Time series of the fluctuating lift coefficient $C_{\mathrm{L}}^{\prime}$ (first column), PSD of the fluctuating lift coefficient (second column) and phase portraits (third column) for the cylinder forced to oscillate at $(A, F)=(1.00,0.90)$ and $(A, F)=(1.25,1.50)$.

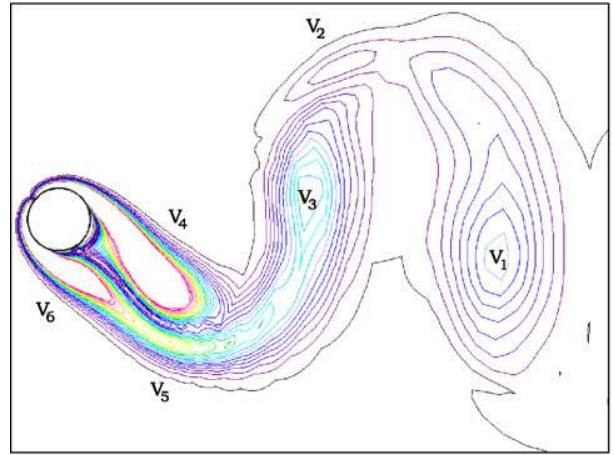

(a) 2P-like vortex shedding mode

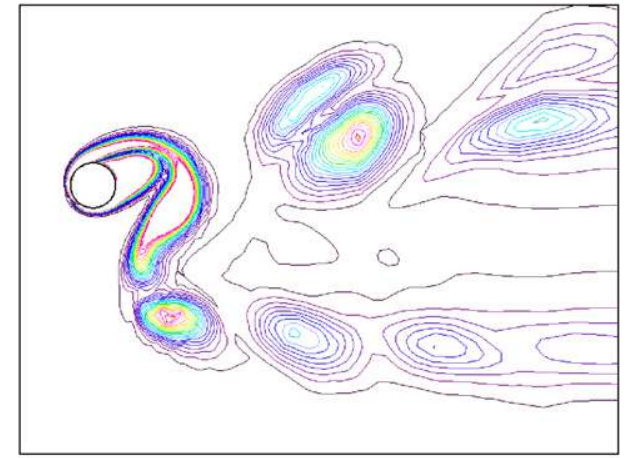

(b) $\mathrm{P}+\mathrm{S}$ vortex shedding mode

Fig. 14. Different vortex shedding modes obtained for $(A, F)=(1.00,0.90)$ (a) and $(A, F)=(1.25,1.50)$ (b).

is not surprising to obtain a deformed shedding mode instead of the regular 2P mode. As already mentioned, the reason comes certainly from the low Reynolds number used here. Indeed, it has been noted by Khalak and Williamson [22] that the 2P mode is not observed at low Reynolds numbers but the reasons why the wake is different remain obscure. Experiments presented by Ramberg and Griffin [37] exhibited only the $\mathrm{P}+\mathrm{S}$ mode for $R e<190$; the $2 \mathrm{P}$ mode in their laminar-regime studies has nonetheless never been observed.

The couple $(A, F)=(1.25,1.50)$ located in the middle of the $\mathrm{P}+\mathrm{S}$ shedding mode area on the shedding mode map, leads to a wake composed of two distinct rows of vortices. In the upper row, the vortices are grouped by pairs $(\mathrm{P})$ whereas a single vortex (S) is shed in the lower row. The wake pattern is shown in Fig. 14b. The time evolution of the fluctuating lift coefficient $C_{\mathrm{L}}^{\prime}$ and the frequency content can be linked to the vortex shedding regimes. Times series for the two cases $(A, F)=(1.00,0.90)$ and $(A, F)=$ $(1.25,1.50)$ (Fig. 13, col. 1) do not exhibit any beating behavior but the signal is modulated by a second frequency over one cycle of oscillation. These additional frequencies appear on the PSD spectra shown in Fig. 13, col. 2. The main difference between the preceding cases at $A=0.25$ 
is that these frequencies are now high frequencies which do not affect the cycle-to-cycle periodicity but only the shape of each cycle. The phase portraits (Fig. 13, col. 3) therefore exhibit only one path but the shape is not ovoid like for locked configurations because of the presence of additional frequencies. The question here is to determine whether the wake is locked or not, since the spectra contains more than one frequency but the phase portraits exhibit only one path. It could be argued that although several frequencies exist, the wake is locked since the main frequency is always at $f^{*}=1.0$ and the additional high frequencies do not affect the cycle-to-cycle periodicity. The major difference between the present cases and the preceding (at $F=0.25$ ) concerns the position of the second peak relative to the first. For a small amplitude of oscillation $A=0.25$, the second peak is a low-frequency peak. As a result, a low-frequency beating behavior is observed: the fluctuations of the lift are not periodic over one cycle of oscillation but over several ones. On the contrary, for high amplitudes $(A=1.25$ and $A=1.00$ ), the secondary peak in the spectrum is a high frequency peak. The fluctuations of the lift are therefore modulated by a high frequency signal which influences the response during one cycle. The fluctuations of the lift remain thus periodic between two successive cycles, but over one cycle, a small fluctuation is observed. We suppose that this fluctuation could be related to the emission of the pair in the upper side of the wake.

The influence of the amplitude on the vortex shedding mode is crucial. Indeed, according to the values, high- or low-frequency phenomena are observed. For small amplitudes, the low-frequency beating behavior in the time histories of $C_{\mathrm{L}}^{\prime}$ has been linked by Placzek et al. [35] to a vortex merging in the wake for $(A, F)=(0.25,1.25)$. On the contrary, for high amplitudes $((A, F)=(1.00,0.90)$ and $(A, F)=(1.25,1.50))$, the appearance of high frequencies in the spectra leads to the emission of a pair of vortices in the upper side of the wake, which could be related to the modulation observed in the time evolution of the lift coefficient.

This first work has shown that the phenomena commonly observed in the case of a cylinder forced to oscillate in a transverse flow can be reproduced with our industrial code. In view of the preceding results, the simulation of vortex-induced vibrations seems to be feasible and results for $R e=100$ are presented in the sequel.

\section{Vortex-induced vibrations}

The case of an elastically mounted cylinder vibrating as a result of fluid forcing is one of the most basic and revealing cases in the general subject of bluff-body fluid-structure interactions. The first part of the work has been conducted with the aim of illustrating and understanding the phenomena involved when the cylinder was subjected to forced oscillations whose characteristics were known, as well as demonstrating the ability of the CFD code to capture the physics of VIV. Similar phenomena are now observed when the vibrations are induced by the flow, the frequency and amplitude responses being in this case not known $a$ priori.

The vortex shedding process in the wake leads to fluctuating drag and lift forces which cause the oscillations of the cylinder. The phenomenon is self-limited: the fluid flow adjusts so that the oscillation amplitude is restricted to a certain upper limit. It has been observed by Mittal and Kumar [27] that the various mechanisms by which the oscillator is able to self-limit its vibration amplitude are a reduction in the amplitude of the aerodynamic forces, appearance of additional frequency components in the time histories of the fluid forces and de-tuning of the vortexshedding frequency from the structural frequency. Although the phenomenon has been observed for a long time, the maximal amplitude of vibration is not yet clearly defined. Indeed, the cylinder response depends on various parameters and particularly the mass-damping parameter $m^{*} \zeta$, where $m^{*}$ represents the adimensional cylinder mass and $\zeta$ is the structural damping. Khalak and Williamson give in their article the schematic amplitude response of the cylinder for high or low mass-damping parameters (see Fig. 3 of [22]). For high $m^{*} \zeta$, only two branches (called initial excitation branch and lower branch) are observed, whereas the behavior at low $m^{*} \zeta$ is more complex and involves three branches (namely the two preceding branches and an additional one called the upper branch). Each branch of the response characterizes a different regime, where the adimensional amplitude of oscillation $A$ is more or less pronounced. The determination of the maximal amplitude that can be reached is still an open question: while the maximal amplitude on the lower branch seems to be about $A=0.6$, the maximum reached on the upper branch is highly dependent on the mass-damping parameter. Khalak and Williamson suggest $A=1.20$ as the maximal value but they find no signs of amplitude saturation when the mass-damping parameter was reduced to extremely small values.

For hydrodynamic applications like ours, the massdamping parameter takes generally low values and the cylinder response should exhibit three branches. However $m^{*} \zeta$ is not only responsible for the number of branches in the amplitude response. There is clearly an influence of the Reynolds number, especially at low values like in the laminar shedding regime $(R e \approx 100)$. Attention has been drawn before for forced oscillations on the fact that the $2 \mathrm{P}$ shedding mode was not observed at low Reynolds number. The differences in the vortex shedding mode also exist for VIV and they prohibit the jump to the upper branch. This is confirmed by the $2 \mathrm{D}$ direct numerical simulation data from Newman and Karniadakis [28] for $R e=100$ and 200 and the low $R e$ experiments of [4] which did not show the upper branch. The absence of the upper branch in the simulations may be due to the fact that the vortex shedding mode obtained at low $R e$ does not give a net energy transfer from the fluid to body motion over one cycle, unlike the $2 \mathrm{P}$ mode at higher $R e$ [22]. 
The numerical results exposed in the following will be compared to those of Shiels et al. [40] who also carry out simulations at $R e=100$. Indeed the main part of their results is presented in the case of an undamped oscillator like in the present study. To begin with the VIV study, the various non dimensional parameters commonly used to classify the response regimes of the cylinder are briefly reminded before turning to numerical results. Then the results of our simulations for an undamped cylinder at $R e=100$ are presented and compared to those of Shiels et al.

\subsection{Adimensional parameters}

The mass-damping parameter $m^{*} \zeta$ is adapted to determine whether the cylinder response is composed of two or three branches, but for a given response shape ( 2 or 3 branches), it remains to determine the best parameter which collapses the different response regimes for a large range of structural parameters. The reduced velocity $U^{*}$ (typically a reference velocity like $U_{\infty}$ divided by the cylinder diameter and a frequency like $f_{\mathrm{N}}$ for example) has often been used to plot the results but the width of the amplitude peak varies with the mass of the system. A "true" reduced velocity $U^{*} / f^{*}$ used by Khalak and Williamson [22] seems to collapse ideally the results over a wide range of cylinder mass. The adimensional parameters used in different studies are thus quite diversified because of the different techniques employed to make the cylinder equation adimensional. The general equation of the oscillator with structural damping is:

$m \ddot{y}+c \dot{y}+k y=F_{y}$

where $m$ is the cylinder mass, $c$ is the structural damping, $k$ is the rigidity and $F_{\mathrm{y}}$ is the resultant of the lift force. The natural frequency $f_{\mathrm{N}}$ of the cylinder (see Eq. (5)) and the frequency in water $f_{\mathrm{H}}$ (defined below Eq. (12)) are often used in the adimensionalization process:

$f_{\mathrm{H}}=\frac{1}{2 \pi} \sqrt{\frac{k}{m+m_{\mathrm{a}}}}$ where $m_{\mathrm{a}} \approx m_{\mathrm{d}}=\rho \pi R^{2} L$

The frequency in water $f_{\mathrm{H}}$ depends on the added mass $m_{\mathrm{a}}$ which can be approximated here by the displaced mass of water $m_{\mathrm{d}}$ as the cylinder is situated in an infinite domain where the viscosity is small. Since no universal set of adimensional parameters exists, the comparison of the results between them requires to juggle with those used in different studies. For that purpose, Table 1 summarizes the different sets of adimensional parameters commonly used in the literature and to which we will refer in the following to compare our results.

The set of parameters used by Shiels et al. [40] (see the last column of Table 1) have been especially developed to by-pass the problem of definition for the structural frequencies $f_{\mathrm{N}}$ and $f_{\mathrm{H}}$ in extreme cases where $k$ and/or $m$ are null. A complementary adimensional rigidity
Table 1

Adimensional sets of parameters commonly used to classify the VIV response regimes

\begin{tabular}{llll}
\hline $\begin{array}{l}\text { Adimensionnal } \\
\text { parameter }\end{array}$ & $\begin{array}{l}\text { Guilmineau and } \\
\text { Queutey [18] }\end{array}$ & $\begin{array}{l}\text { Khalak and } \\
\text { Williamson [22] }\end{array}$ & $\begin{array}{l}\text { Shiels et al. } \\
{[40]}\end{array}$ \\
\hline Amplitude $A^{*}$ & $y / d$ & $y / d$ & $y / d$ \\
Frequency $f^{*}$ & $f / f_{\mathrm{N}}$ & $f / f_{\mathrm{H}}$ & $f d / U_{\infty}$ \\
Velocity $U^{*}$ & $U_{\infty} /\left(f_{\mathrm{N}} d\right)$ & $U_{\infty} /\left(f_{\mathrm{H}} d\right)$ & $U_{\infty} /\left(2 \pi f_{\mathrm{N}} d\right)$ \\
Mass $m^{*}$ & $m / m_{\mathrm{d}}$ & $m / m_{\mathrm{d}}$ & $m /\left(0.5 \rho d^{2} L\right)$ \\
Damping $\zeta$ & $c / \sqrt{k m}$ & $c / \sqrt{k\left(m+m_{\mathrm{d}}\right)}$ & $c /$ \\
& & & $\left(0.5 \rho U_{\infty} d L\right)$ \\
\hline
\end{tabular}

$y$ is the vertical cylinder displacement, $d$ is the cylinder diameter and $L$ its length, $U_{\infty}$ is the inlet velocity and $\rho$ is the density of the fluid, $m, k$ and $c$ are respectively the cylinder mass, rigidity and damping, $f$ is the actual oscillation frequency of the cylinder, $f_{\mathrm{N}}$ is the natural cylinder frequency Eq. (5), $f_{\mathrm{H}}$ is the natural frequency in water Eq. (12) and finally $m_{\mathrm{d}}$ is the displaced mass of water Eq. (12).

$k^{*}=k /\left(0.5 \rho U_{\infty}^{2} L\right)$ is also introduced by Shiels et al. [40] to define a new representative parameter $k_{\text {eff }}^{*}$ called the "effective rigidity":

$k_{\mathrm{eff}}^{*}=k^{*}-4 \pi^{2} f^{* 2} m^{*}$

This parameter is always defined, even if the mass or the rigidity (or both) are null. Moreover, it collapses very well the data for different structural parameters.

\subsection{Response of the cylinder undergoing VIV}

A set of simulations is performed without structural damping and thus the movement is governed by Eq. (4). The time integration is realized with the blended procedure (see Eqs. (6)-(8)), but further simulations are currently carried out with the implicit procedure already employed by Abouri [1] and Sigrist and Abouri [42]. The behavior of the cylinder is summarized on the curves plotted in Fig. 15. The response regimes for the amplitude of oscillation, the actual frequency of the cylinder and the aerodynamic coefficients are plotted against $k_{\text {eff }}^{*}$.

As expected, the amplitude response is only composed of the lower branch. The results show a very good agreement with those of Shiels et al. [40], for each parameter. A "resonant" zone is observed in the range $k_{\mathrm{eff}}^{*} \approx[0-5]$, where the maximal amplitude of oscillation reaches $A=0.58$ at $k_{\text {eff }}^{*}=2.32$ (Fig. 15a). The peak of amplitude is naturally associated with an increase of the aerodynamic coefficients. Fig. 15b presents the evolution of the actual reduced frequency of the cylinder $f^{*}=f d / U_{\infty}$. Inside the "resonant" zone, the frequency shifts from the Strouhal frequency $f_{\mathrm{S}}$ and increases until a value corresponding to the natural frequency of the cylinder: the "resonant" zone is thus similar to the lock-in zone described previously for forced vibrations and the same name is therefore adopted to describe this zone. Finally, the mean drag and maximal lift coefficients are characterized by a maximum inside the lock-in zone which is associated to the maximal amplitude response (Fig. 15c). Outside this zone, the aerodynamic 


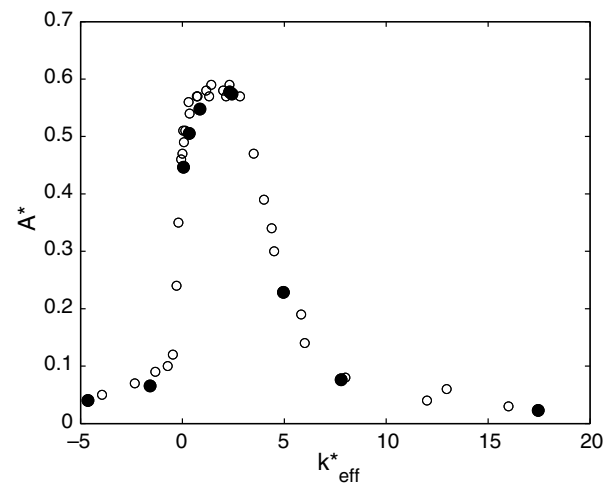

(a) Amplitude response

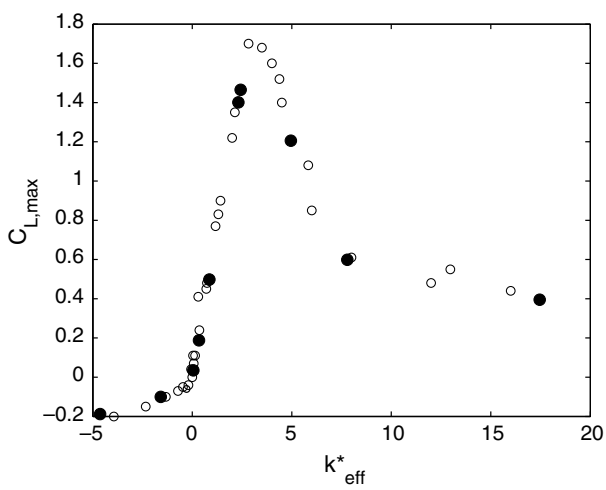

(c) Lift coefficient response

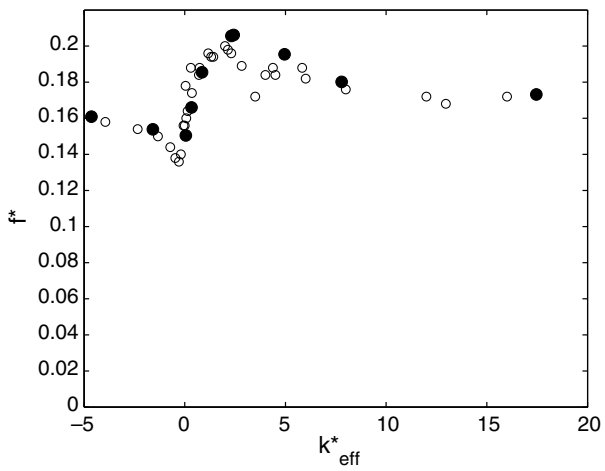

(b) Frequency response (actual frequency)

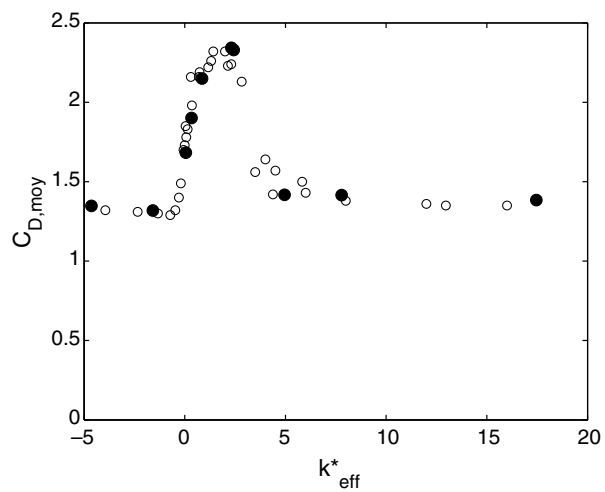

(d) Drag coefficient response

Fig. 15. Computed response (๑) of the cylinder (amplitude $A$ (a), actual frequency $f^{*}=f_{\mathrm{D}} / U_{\infty}$ (b), maximal lift coefficient $C_{\mathrm{L}, \max }^{\prime}$ (c) and mean $\operatorname{drag}$ coefficient $\left.\overline{C_{\mathrm{D}}^{\prime}}(\mathrm{d})\right)$ compared to the results of $[40](\bigcirc)$.

coefficients relapse near the values corresponding to the fixed cylinder case. Values of the lift for $k_{\text {eff }}^{*}<0$ have been set according to Shiels et al. [40] to the opposite of the $C_{\mathrm{L}, \max }^{\prime}$ value in order to indicate the existence of a phase shift equal to $\pi$ between the cylinder displacement and the $C_{\mathrm{L}}^{\prime}$. Without this artefact, the curve would have had the same shape as the one of the reduced frequency $f^{*}$. In the following, a detailed analysis of three cases $\left(k_{\mathrm{eff}}^{*}=17.5, k_{\mathrm{eff}}^{*}=2.32\right.$ and $\left.k_{\mathrm{eff}}^{*}=0.05\right)$ is exposed : the frequency content, vortex shedding modes and lock-in zone are studied and the phenomena observed are linked together.

\subsubsection{Frequency content and phase portraits}

Time histories of the cylinder displacement are now very interesting data, because the motion is not known a priori as it was the case for forced vibrations. The graphs in the first row Fig. 16 highlight the difference of amplitude between the different regimes for the cylinder displacement. In the lock-in zone, the amplitude is maximal but remains limited. This is due to the self-limitation phenomenon mentioned before. The periodic regime is reached quickly: about 10 oscillation cycles only are necessary to reach the maximal amplitude response. The PSDs of the fluctuating lift coefficient $C_{\mathrm{L}}^{\prime}$ are plotted versus the frequency ratio $f^{*}=f / f_{\mathrm{H}}$ in the second row Fig. 16. For the intermediate case $k_{\mathrm{eff}}^{*}=2.32$, the peak at $f^{*} \approx 1.0$ indicates that the lift is governed by the natural frequency of the structure instead of the Strouhal frequency $f_{\mathrm{S}}$ for $R e=100$. The adimensional frequencies $f^{*}=f_{\mathrm{S}} / f_{\mathrm{H}}$ corresponding to the Strouhal frequency at $R e=100$ in the three cases are: $f^{*}=1.35$ for $k_{\text {eff }}^{*}=0.05, f^{*}=0.82$ for $k_{\mathrm{eff}}^{*}=2.32$, and $f^{*}=0.51$ for $k_{\mathrm{eff}}^{*}=17.5$. The preceding values indicate that for $k_{\mathrm{eff}}^{*}=0.05$ and $k_{\mathrm{eff}}^{*}=17.5$, the oscillations of the cylinder are driven by the Strouhal frequency, as the peaks highlighted in Fig. 16 are located approximately at $f^{*}=f_{\mathrm{S}} / f_{\mathrm{H}}$. On the contrary, the spectrum for $k_{\mathrm{eff}}^{*}=2.32$ reveals that the lift is governed by the structural frequency $f_{\mathrm{H}}$ since the peak is located at $f^{*} \approx f / f_{\mathrm{H}}=1.0$.

Over a certain range of $k_{\text {eff }}^{*}$, the cylinder response exhibits an amplification of the amplitude and of the aerodynamic coefficients. This amplification can be related to the synchronization of the cylinder frequency on its natural frequency: this is typical for the lock-in zone, which is defined as the domain where the cylinder frequency shifts from the Strouhal frequency and locks on the structural one.

The phase portraits of the oscillator are represented on the third row Fig. 16. Unlike the forced vibration case, the shape of the phase portraits is similar for each $k_{\mathrm{eff}}^{*}$ either inside or outside the lock-in zone. The limit cycle is no longer ovoid but always resembles a double Lissajou figure. Mittal and Kumar [27] observed a simple Lissajou figure in the case of in-line and transverse free oscillations but the 

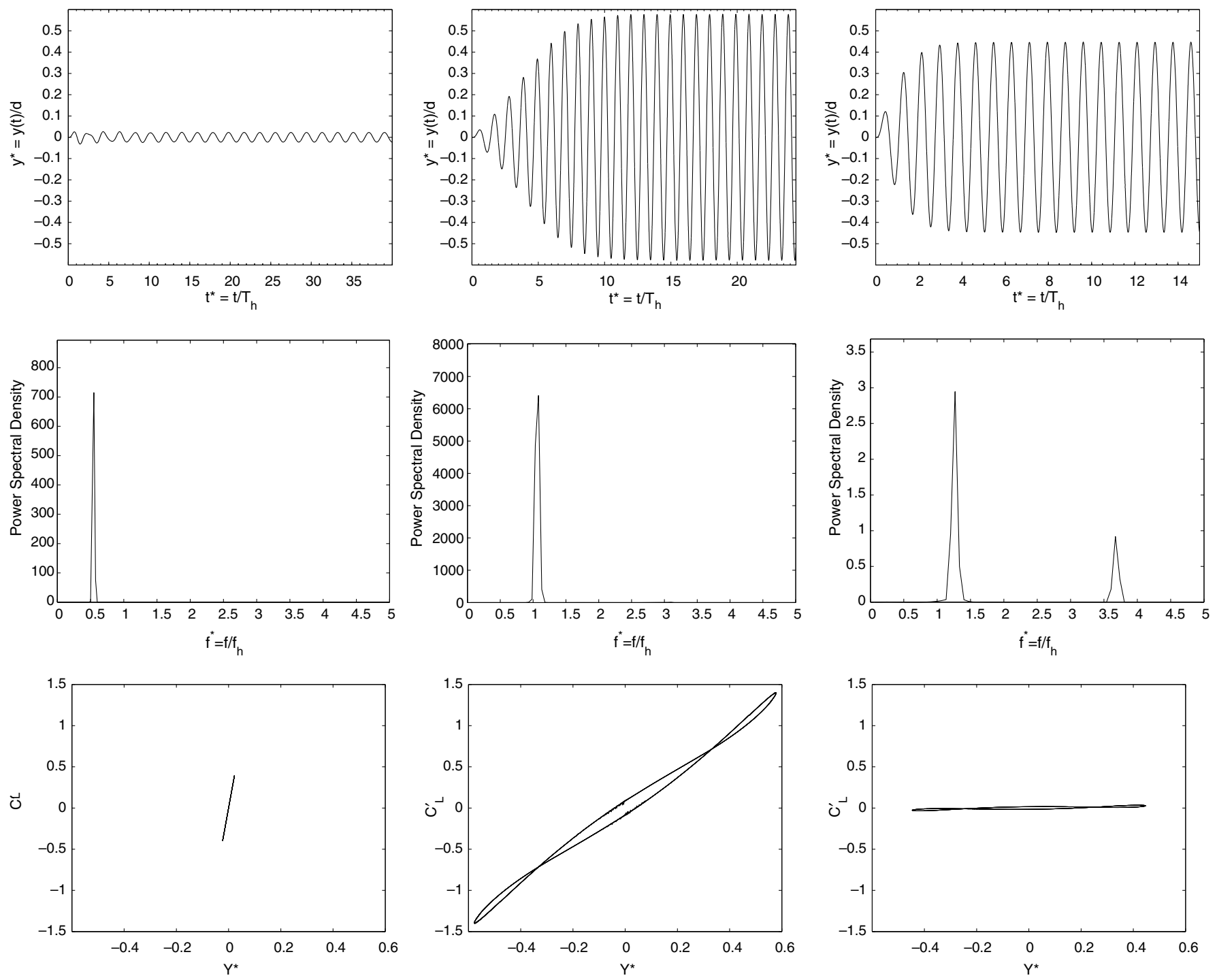

Fig. 16. Time histories of the cylinder displacement (first row), PSDs of the lift coefficient (second row) and phase portraits (third row) for VIV at $R e=100$. From left to right: $k_{\mathrm{eff}}^{*}=17.5,2.32$ and 0.05 .

same shape of phase portrait exhibiting a double Lissajou has also been obtained by Guilmineau and Queutey [18] for transverse oscillations like here. The three phase portraits of Fig. 16 can be compared to those plotted by Khalak and Williamson [22], see Fig. 13 in this reference. Firstly, the global inclination of the phase portraits becomes progressively horizontal like here as $k_{\text {eff }}^{*}$ decreases (or equivalently as $U^{*}$ increases). Secondly, the intermediate case located in the lock-in zone is obviously more regular in this study as the upper branch is not reached. Indeed, Khalak and Williamson noted that the irregularities appearing in the phase portrait were the manifestation of the less steady dynamics of the upper branch. Finally, the extent of the first and last portraits is like in our case smaller than for the intermediate case. The absence of the upper branch in our case also explains why the global inclination of the portrait is always in the first quadrant for the three cases: Khalak and Williamson [22] obtained an incli- nation in the second quadrant for the last case because a phase jump occurred. One reason for the absence of phase jump in our study can be the low Reynolds number used $[18,22]$, which prohibits on the one hand the modification of the vortex shedding modes as it will be seen later and on the other hand the jump to the upper branch.

\subsubsection{Vortex shedding modes}

The vortex shedding patterns for the three cases under study are illustrated in Fig. 17 with the vorticity field. The vortices are shed alternately from the upper and lower sides of the cylinder, according to the classical Von Kármán streets or $2 \mathrm{~S}$ mode: no clear modification of the wake has been observed over the range of $k_{\mathrm{eff}}^{*}$ studied. The vortices are more stretched in the vertical direction at $k_{\mathrm{eff}}^{*}=2.32$ than in the other cases. This slight modification is certainly due to the increased amplitude of oscillation in the intermediate case, which deforms the wake in the verti- 


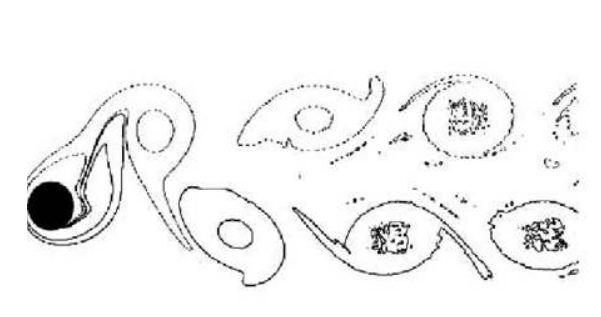

(a) $k_{\text {eff }}^{*}=2.31$ (from Shiels et al. (2001))

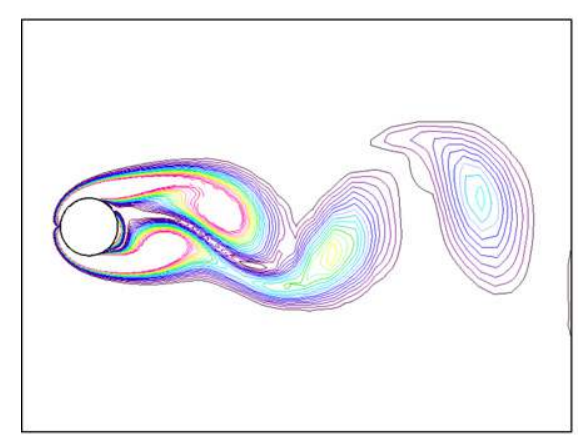

(c) $k_{\text {eff }}^{*}=17.5$

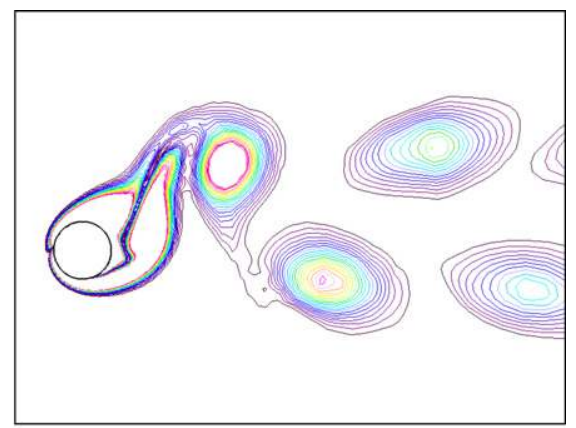

(b) $k_{\text {eff }}^{*}=2.32$

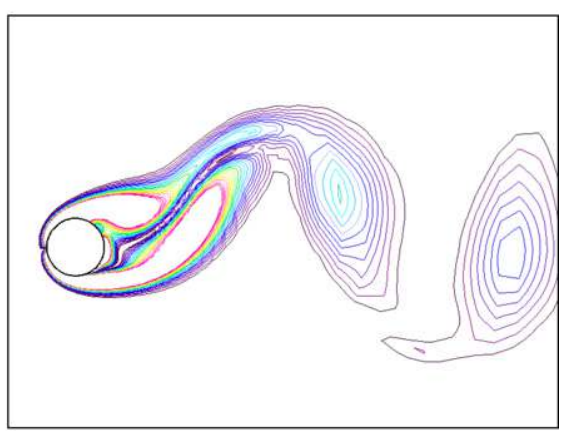

(d) $k_{\text {eff }}^{*}=0.05$

Fig. 17. Vortex shedding modes inside and outside the lock-in zone for the cylinder undergoing VIV at $R e=100$.

cal direction. Similar results have been obtained by Shiels et al. [40], as it can be seen in Fig. 17a.

For higher Reynolds numbers, a change between a $2 \mathrm{~S}$ and $2 \mathrm{P}$ mode is often observed $[22,18]$ and can be linked to the transition between the upper and lower branch of excitation. This transition has also been connected to the phase jump in the case of forced oscillations or VIV. However, at low Reynolds number such a change of the mode shape is not observed. Moreover, the numerical simulations fail sometimes to capture this mode change, even for high Reynolds numbers: in their study Al-Jamal and Dalton [2] did not obtain the $2 \mathrm{~S}$ and $2 \mathrm{P}$ modes although they use large eddy simulations at $R e=8000$. The authors argue that these modes are only observed for sinusoidal oscillations like those used in forced oscillations. When oscillations are not purely sinusoidal, as it is the case in VIV for certain reduced velocities, the $2 \mathrm{~S}, 2 \mathrm{P}$, etc. structures may not become fully established because the amplitude of oscillation and phase angle are both time dependent. The lack of constancy in amplitude and phase angle could likely lead to the lack of repeatability in vortex formation which certainly could suppress the standard mode patterns.

The oscillations of the cylinder are not always purely sinusoidal and a beating behavior similar to the one observed for forced vibrations is observed for example at $k_{\text {eff }}^{*}=7.79:$ the vertical displacement is not periodic between two successive cycles (see Fig. 18a), but over sev-
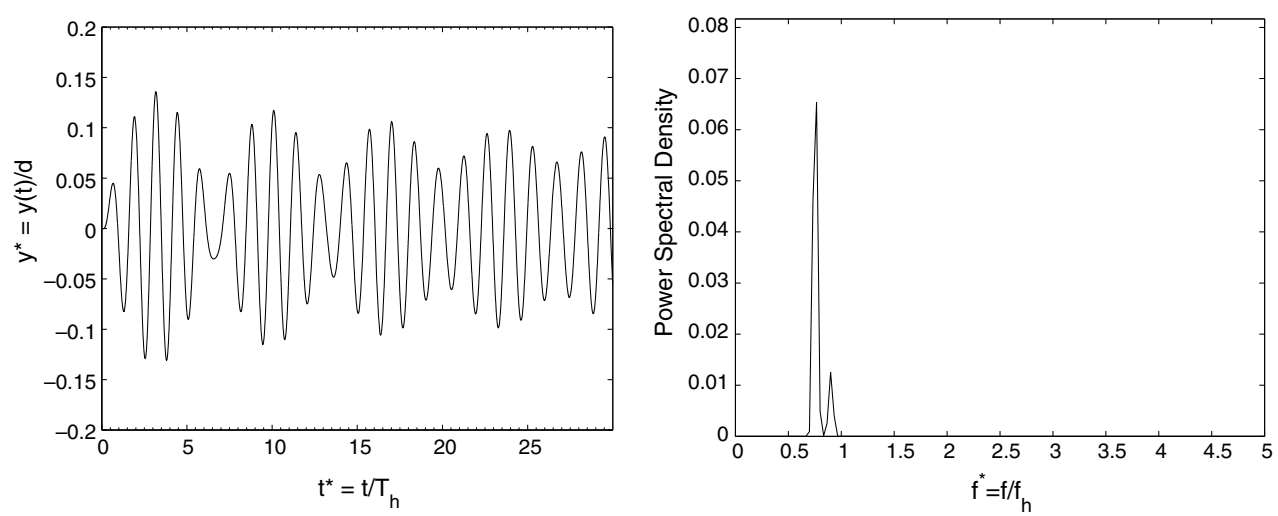

Fig. 18. Desynchronization of the cylinder oscillations at $k_{\mathrm{eff}}^{*}=7.79$ : the peaks of frequency progressively shift from the values $f^{*}=f_{\mathrm{S}} / f_{\mathrm{H}}=0.68$ and $f^{*}=1$. 
eral cycles. The spectrum (Fig. 18b) exhibits therefore two peaks: the main peak is located at $f_{\mathrm{m}}^{*}=0.77$ and the secondary peak at $f_{\mathrm{s}}^{*}=0.90$. It should be noted that these two peaks are between $f^{*}=f_{\mathrm{S}} / f_{\mathrm{H}}=0.68$ and $f^{*}=f_{\mathrm{H}} / f_{\mathrm{H}}=1$, the first value corresponding to the Strouhal frequency whereas the second is the natural cylinder frequency in water. The beating behavior can thus be understood as a desynchronization phenomenon: the cylinder oscillation frequency progressively shifts from the natural frequency $f_{\mathrm{H}}$, which is predominant at $k_{\mathrm{eff}}^{*}=2.32$, to the Strouhal frequency which drives the wake at $k_{\mathrm{eff}}^{*}=17.5$. When $k_{\mathrm{eff}}^{*}$ is increased, there is a progressive change of the main peak in the spectrum associated with the end of the lock-in zone and which is the sign of the response modification.

This beating behavior is often observed $[22,27,40,2]$ and has been related to a mode change, or a mode competition. Khalak and Williamson [22] have shown that this beating is associated which the transition between the lower and upper branch of excitation, and therefore with the transition between the $2 \mathrm{~S}$ and $2 \mathrm{P}$ modes. We do not have a significant mode change, but the beating is however connected to the little modification of the vortex patterns between $k_{\text {eff }}^{*}=2.32$ and $k_{\text {eff }}^{*}=17.5$. As mentioned above, at low Reynolds number the classical mode change $2 \mathrm{~S}$ to $2 \mathrm{P}$ is not observed; however, a little modification of the wake appears and a similar beating phenomenon happens, which is related to this vortex pattern change and to the progressive transition between a cylinder response driven by the natural frequency $f_{\mathrm{N}}$ to a response characterized by the Strouhal frequency $f_{\mathrm{S}}$.

\subsubsection{Lock-in zone}

The lock-in zone can be identified in Fig. 15a as the region where an amplification of the oscillation amplitude is observed. This first representation focuses on the amplitude resonance which characterizes the lock-in. A second interpretation (Fig. 15b) consists in defining the lock-in as the range where the frequency shifts from the Strouhal value. Instead of plotting the reduced frequency $f^{*}=f_{\mathrm{D}} / U_{\infty}$, the lock-in can also be characterized by representing the adimensional frequency $f^{*}=f / f_{\mathrm{H}}$ against a representative parameter (the effective rigidity $k_{\text {eff }}^{*}$ like [40] or the reduced velocity $U^{*}$ like [22]). In this case, the lock-in zone can be interpreted as the region where the actual cylinder oscillation frequency $f$ is approximately equal to the natural cylinder frequency $f_{\mathrm{H}}$. This approach is represented in Fig. 19. As there is no evident relation between the effective rigidity $k_{\mathrm{eff}}^{*}$ used by Shiels et al. [40] and the reduced velocity $U^{*}$ adopted by Khalak and Williamson [22], the subplot in Fig. 19 gives a relation in the form of a curve to facilitate the link with the previous paragraphs.

The horizontal line $f^{*}=1$ indicates that the cylinder oscillation frequency is the natural frequency, while the oblique lines give the Strouhal frequencies over the range of reduced velocities studied. Two lines are represented because results from Khalak and Williamson have been

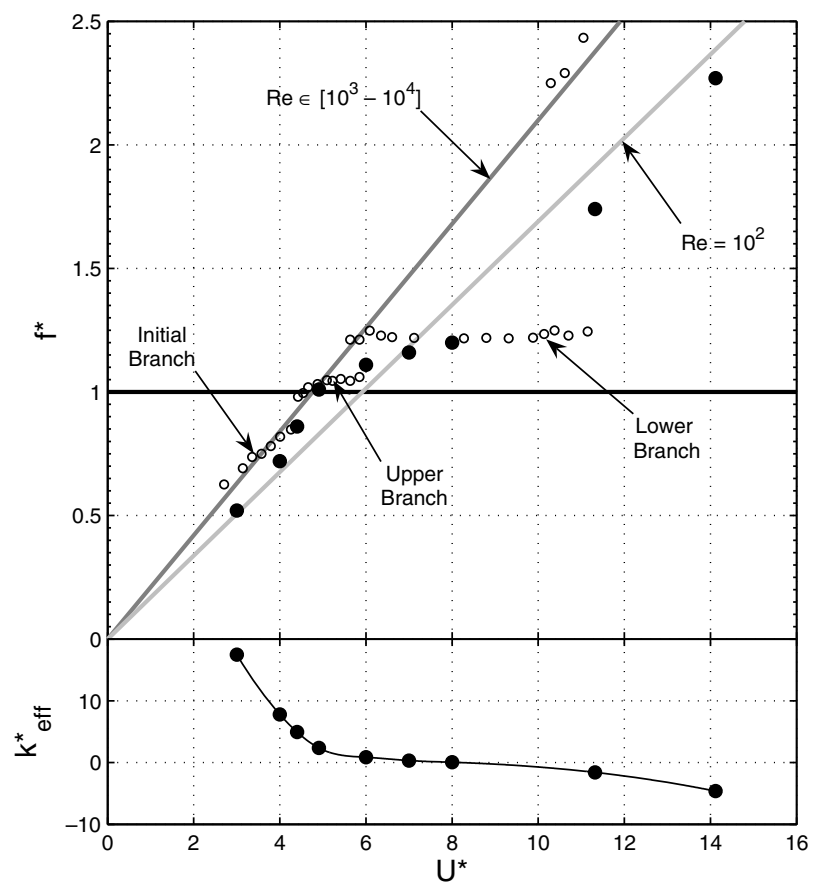

Fig. 19. Reduced frequency response $f^{*}=f / f_{\mathrm{H}}$ and lock-in zone. ( Present simulation, $(\bigcirc)$ [22]. The black line indicates the oscillation at $f_{\mathrm{H}}$ whereas the gray lines correspond to oscillations at the Strouhal frequency for the range of Reynolds number considered. The subplot give the correspondence between the parameters $k_{\mathrm{eff}}^{*}$ and $U^{*}$ used respectively by Shiels et al. [40] and Khalak and Williamson [22].

obtained at higher Reynolds numbers for which the Strouhal number is slightly greater (the results of Khalak and Williamson [22] spread over the interval $\operatorname{Re} \approx\left[10^{3} ; 10^{4}\right]$ where fortunately the Strouhal number is nearly constant and will be approximated here by the average value $S t=0.21)$.

If we observe first the results of Khalak and Williamson [22], it can be seen that for small or high $U^{*}$, the cylinder oscillations are driven by the Strouhal. In the range $U^{*} \approx[4-6]$, the cylinder frequency $f$ is exactly equal to the natural frequency of the cylinder: this corresponds to the upper branch. Over the range $U^{*} \approx[6-10]$, the frequency $f$ is still not equal to the Strouhal frequency but its value remains constant over this interval: this corresponds to the lower branch. The interval $U^{*} \approx[4-10]$ can be defined as the lock-in zone because the cylinder frequency shifts from the Strouhal frequency. The transition zone between the upper and lower branch is characterized by the simultaneous existence of two frequencies in the response.

In our case, the response is less complex because only one branch is observed. However, the two first and last points $\left(U^{*}=3.0,4.0\right.$ and $\left.U^{*}=11.3,14.1\right)$ show that the cylinder oscillations are in this case driven by the Strouhal frequency. In the range $U^{*} \approx[4.4-11.3]$, the actual frequency of the cylinder shifts progressively from the Strouhal frequency and follows an oblique line whose slope is very smooth and quasi-horizontal. Over this interval, the cylinder is locked near the natural frequency of the cylinder. In the present case, the lock-in zone is not strictly 
horizontal but rather oblique. This is due to the mass of the system as it is shown by Shiels et al. [40]. The authors perform several series of simulations for different mass ratios $m^{*}$ between 0 and 20 . For small masses, the lock-in zone is not very pronounced. On the contrary, for high mass ratios the lock-in zone is immediately visible and forms a horizontal level located at $f^{*}=1$ (see Fig. 13 of [40]). In most cases tested here, we choose a mass ratio $m^{*}=3.3$, which explains the slight slope of the level.

\section{Conclusion}

The numerical simulation of the vortex-induced vibrations phenomenon has been investigated here at $R e=100$. The first task of the study has shown that a rather accurate description of the wake for a fixed cylinder could be obtained. Based on these results, the simulation of a cylinder forced to oscillate in a transverse stream has been performed to analyze the different phenomena appearing. The frequency content and vortex shedding modes have been studied and linked together by changing the frequency and amplitude of the imposed oscillations. The well-known lock-in zone has been highlighted and characterized with the analysis of PSDs and phase portraits of the cylinder displacement and lift coefficient.

This step has provided interesting elements to understand the phenomena involved and to validate the CFD code: the numerical results have indeed proved that our industrial code was able to capture the vortex induced vibration phenomenon. The lock-in zone has been characterized and the response regimes (amplitude, actual oscillation frequency and aerodynamic coefficients) are in good agreement with similar studies. Restrictions must however be underlined as the results are for instance in good agreement for low values of the Reynolds number and without structural damping. Ongoing numerical simulations are performed for higher Reynolds number $(1000 \leqslant R e \leqslant 10.000)$ with turbulence modeling using the $\mathrm{k}-\omega \mathrm{SST}$ model of Menter [26]. The results are rather promising: the amplitude response is correctly evaluated for high reduced velocities and the upper branch is reached [43]; these results are therefore very encouraging since previous attempts to simulate the upper branch with numerical approaches somehow failed to predict the upper branch response [18,31].

Detailed investigation and analysis of these complementary simulations will be presented in a coming paper and future application of the presented work will be devoted to numerical simulation of flow-induced vibration phenomenon in tube bundle of nuclear propulsion steam generators or vortex-induced vibrations on mooring cables and periscopes.

\section{References}

[1] Abouri D. Un algorithme d'interaction Fluide-Solide Rigide: Application à la Volumétrie. Ph.D. thesis, Université de Poitiers; 2003.
[2] Al-Jamal H, Dalton C. Vortex induced vibrations using large eddy simulation at a moderate Reynolds number. J Fluids Struct 2004;19(1):73-92.

[3] Anagnostopoulos P. Numerical study of the flow past a cylinder excited transversely to the incident stream. Part 1: Lock-in zone, hydrodynamic forces and wake geometry. J Fluids Struct 2000;14(6):819-51.

[4] Anagnostopoulos P, Bearman P-W. Response characteristics of a vortex-excited cylinder at low Reynolds numbers. J Fluids Struct 1992;6(1):39-50.

[5] Braza M, Chassaing P, Ha Minh H. Numerical study and physical analysis of the pressure and velocity fields in the near wake of a circular cylinder. J Fluid Mech 1986;165:79-130.

[6] Breuer M. A challenging test case for large eddy simulation: high Reynolds number circular cylinder flow. Int $\mathbf{J}$ Heat Fluid Flow 2000;21(5):648-54.

[7] Carberry J, Sheridan J, Rockwell D. Forces and wake modes of an oscillating cylinder. J Fluids Struct 2001;15(3-4):523-32.

[8] Chen S-S. Flow-induced vibration of circular cylindrical structures. Springer Verlag; 1987.

[9] Dennis S-C-R, Chang G-Z. Numerical solutions for steady flow past a circular cylinder at low Reynolds numbers up to 100. J Fluid Mech 1970;42:471-89.

[10] Donea J, Guiliani S, Halleux J-P. An arbitrary Lagrangian-Eulerian finite element method for transient dynamic fluid-structure interactions. Comput Methods Appl Mech Eng 1982;33(1-3):689-723.

[11] Dong S, Karniadakis G-E. DNS of flow past a stationary and oscillating cylinder at $R e=10000$. J Fluids Struct 2005;20(4): 519-31.

[12] Farhat C, Lesoinne M, Stern P. High performance solution of threedimensional nonlinear aeroelastic problems via parallel partitioned algorithms: methodology and preliminary results. Adv Eng Software 1997;28(1):43-61.

[13] Ferziger J-H, Perić M. Computational methods for fluid dynamics. Springer Verlag; 1996.

[14] Fey U, König M, Eckelmann H. A new Strouhal-Reynolds-number relationship for the circular cylinder in the range $47<R e<2 \cdot 10^{5}$. Phys Fluids 1998;10(7):1547-9.

[15] Fujisawa N, Asano Y, Arakawa C, Hashimoto T. Computational and experimental study on flow around a rotationally oscillating circular cylinder in a uniform flow. J Wind Eng Ind Aerodyn 2005;93(2):137-53.

[16] Gerouache M-S. Étude numérique de l'instabilité de Bénard-Kármán derrière un cylindre fixe ou en mouvement périodique. Dynamique de l'écoulement et advection chaotique. Ph.D. thesis, École Polytechnique de l'Université de Nantes; 2000.

[17] Glück M, Breuer M, Durst F, Halfmann A, Rank E. Computation of wind-induced vibrations of flexible shells and membranous structures. J Fluids Struct 2003;17(5):739-65.

[18] Guilmineau E, Queutey P. Numerical simulation of vortex-induced vibration of a circular cylinder with low mass-damping in a turbulent flow. J Fluids Struct 2004;19(4):449-66.

[19] Henderson R-D. Details of the drag curve near the onset of vortex shedding. Phys Fluids 1995;7:2102-4.

[20] Henderson R-D. Nonlinear dynamics and pattern formation in turbulent wake transition. J Fluid Mech 1997;352:65-112.

[21] Jeon D, Gharib M. On circular cylinders undergoing two-degree-offreedom forced motions. J Fluids Struct 2001;15(3-4):533-41.

[22] Khalak A, Williamson C-H-K. Motions, forces and mode transitions in vortex-induced vibrations at low mass-damping. J Fluids Struct 1999;13(7-8):813-51.

[23] Koobus B, Farhat C. Second-order time-accurate and geometrically conservative implicit schemes for flow computations on unstructured dynamic meshes. Comput Methods Appl Mech Eng 1999;170(1): 103-29.

[24] Koopmann G-H. The vortex wakes of vibrating cylinders at low Reynolds numbers. J Fluid Mech 1967;28(3):501-12. 
[25] Lange C-F, Durst F, Breuer M. Momentum and heat transfer from cylinders in laminar crossflow at $10^{-4} \leqslant R e \leqslant 200$. Int J Heat Mass Transf 1998;41(22):3409-30.

[26] Menter F-R. Two-equation eddy-viscosity turbulence models for engineering applications. AIAA J 1994;32:598-605.

[27] Mittal S, Kumar V. Flow-induced vibrations of a light circular cylinder at Reynolds numbers $10^{3}$ to $10^{4}$. J Sound Vibr 2001;5(245): 923-46.

[28] Newman D, Karniadakis G-E. Simulation of flow over a flexible cable: a comparison of forced and flow-induced vibration. J Fluids Struct 1996;10(5):439-53.

[29] Nobari M-R-H, Naredan H. A numerical study of flow past a cylinder with cross flow and inline oscillation. Comput Fluids 2006;35(4):393-415.

[30] Norberg C. Fluctuating lift on a circular cylinder: review and new measurements. J Fluids Struct 2003;17(1):57-96.

[31] Pan Z-Y, Cui W-C, Miao Q-M. Numerical simulation of vortexinduced vibration of a circular cylinder at low mass-damping using RANS code. J Fluids Struct 2007;23(4):23-37.

[32] Pasquetti R. High-order methods for the numerical simulation of vortical and turbulent flows - high-order LES modeling of turbulent incompressible flow. Comptes Rendus Mécanique 2005;333(1):39-49.

[33] Persillon H, Braza M. Physical analysis of the transition to turbulence in the wake of a circular cylinder by three-dimensional Navier-Stokes simulation. J Fluid Mech 1998;365:23-88.

[34] Piperno S. Simulation Numérique de Phénomènes d'Interaction Fluide-Structure. Ph.D. thesis, École Nationale des Ponts et Chaussées; 1995.

[35] Placzek A, Sigrist J-F, Hamdouni A. Numerical simulation of vortex shedding past a circular cylinder in a cross-flow at low Reynolds number with finite-volume technique. Part 1: forced oscillations. In: 2007 ASME pressure vessels \& piping division conference. ASME, San Antonio (TX), USA; 2007a.

[36] Placzek A, Sigrist J-F, Hamdouni A. Numerical simulation of vortex shedding past a circular cylinder in a cross-flow at low Reynolds number with finite-volume technique. Part 2: flow-induced oscillations. In: 2007 ASME pressure vessels \& piping division conference. ASME, San Antonio (TX), USA; 2007b.
[37] Ramberg S-E, Griffin O-M. Hydroelastic response of marine cables and risers. Hydrodyn Ocean Eng 1981:1223-45.

[38] Roshko A. On the development of turbulent wakes from vortex streets. NACA Report 1191, California Institute of Technology; 1954.

[39] Saghafian M, Stansby P-K, Saidi M-S, Apsley D-D. Simulation of turbulent flows around a circular cylinder using nonlinear eddyviscosity modelling: steady and oscillatory ambient flows. J Fluids Struct 2003;17(8):1213-36.

[40] Shiels D, Leonard A, Roshko A. Flow-induced vibration of a circular cylinder at limiting structural parameters. J Fluids Struct 2001;15(1):3-21.

[41] Sigrist J-F. Modélisation et Simulation Numérique d'un Problème Couplé Fluide/Structure Non Linéaire. Application au dimensionnement de structures nucléaires de propulsion navale. Ph.D. thesis, École Centrale de Nantes - Université de Nantes; 2004.

[42] Sigrist J-F, Abouri, D. Numerical simulation of a non-linear coupled fluid-structure problem with implicit and explicit coupling procedures. In: 2006 ASME pressure vessels \& piping division conference. ASME, Vancouver (BC), Canada; July 2006.

[43] Sigrist J-F, Allery C, Beghein C. A numerical simulation of vortexinduced vibration on a elastically supported rigid cylinder at moderate Reynolds numbers. In: 2008 ASME pressure vessels \& piping division conference. ASME, Chicago (IL), USA; July 2008.

[44] Slone A-K, Pericleous K, Bailey C, Cross M. Dynamic fluidstructure interaction using finite volume unstructured mesh procedures. Comput Struct 2002;80(5-6):371-90.

[45] Socolescu L. Étude numérique de l'instabilité de Bénard-Von Karman derrière un cylindre chauffé. Ph.D. thesis, Université du Havre; 1996.

[46] Star-CD. Star-CD Methodology. CD Adapco Group; 2003.

[47] Tuann S-Y, Olson M-D. Numerical studies of the flow around a circular cylinder by a finite element method. Comput Fluids 1978;6:219-40

[48] Williamson C-H-K. Advances in our understanding of vortex dynamics in bluff body wakes. J Wind Eng Ind Aerodyn 1997:3-32.

[49] Williamson C-H-K, Roshko A. Vortex formation in the wake of an oscillating cylinder. J Fluids Struct 1988;2(4):355-81. 